\title{
Deviation Measures on Banach Spaces and Applications
}

\author{
Christos E. Kountzakis \\ Department of Statistics and Actuarial Financial Mathematics, University of the Aegean, \\ Samos, Greece \\ Email: chr_koun@aegean.gr
}

Received February $6^{\text {th }}, 2013$; revised March $4^{\text {th }}, 2013$; accepted March $11^{\text {th }}, 2013$

\begin{abstract}
In this article we generalize the notion of the deviation measure, which were initially defined on spaces of squarely integrable random variables, as an extension of the notion of standard deviation. We extend them both under a frame which requires some elements from the theory of partially ordered linear spaces and also under a frame which refers to some closed subspace, whose elements are supposed to have zero deviation. This subspace denotes in general a set of risk-less assets, since in finance deviation measures may replace standard deviation as a measure of risk. In the last sections of the article we treat the minimization of deviation measures over a set of financial positions as a zero-sum game between the investor and the nature and we determine the solution of such a minimization problem via min-max theorems.
\end{abstract}

Keywords: Deviation Measure; Expectation-Bounded Risk Measure; Expected Shortfall

\section{Introduction}

Consider two time-periods of economic activity, denoted by 0 and 1 . The time-period 0 is the time-period in which all the individuals make their own decisions under uncertainty, while the time-period 1 is the one in which they enjoy the effects of these decisions, in which the true state of the economy is revealed. Let us consider a Banach space $E$, which is supposed to be the space of financial positions, denoting the total value of a portfolio of assets selected at time-period 0 , when time-period 1 comes. $E$ is usually a space of random variables, namely $E \subseteq L^{0}(\Omega, \mathcal{F}, \mu)$, where $L^{0}(\Omega, \mathcal{F}, \mu)$ is the space of the $\mathcal{F}$-measurable random variables $X: \Omega \rightarrow \mathbb{R}$ defined on the probability space $(\Omega, \mathcal{F}, \mu)$ of the economy, where $\Omega$ denotes the set of states of the world, the $\sigma$-algebra $\mathcal{F}$ denotes the observable events of the economy and $\mu$ denotes a probability measure on the set of events $\mathcal{F}$. We also consider the riskless asset $\mathbf{1}$, being the random variable for which $\mathbf{1}(\omega)=1, \mu$-a.e. . A wedge $P$ of $E$ is a subset of $E$ such that $P+P \subseteq P, \lambda \cdot P \subseteq P$ for any $\lambda \in \mathbb{R}_{+}$. If $P \cap(-P)=\{0\}$ this wedge is called cone.

$P^{0}=\left\{f \in E^{*} \mid f(x) \geq 0\right.$ for any $\left.x \in P\right\}$ is the dual wedge of $P$ in $E^{*}$. Also, by $P^{00}$ we denote the subset $\left(P^{0}\right)^{0}$ of $E^{* *}$. It can be easily proved that if $P$ is a closed wedge of a reflexive space, then $P^{00}=P$. If $P$ is a wedge of $E^{*}$, then the set $P_{0}=\{x \in E \mid \hat{x}(f) \geq 0$ for any $f \in P\}$ is the dual

wedge of $P$ in $E$, where ${ }^{\wedge}: E \rightarrow E^{* *}$ denotes the natural embedding map from $E$ to the second dual space $E^{* *}$ of E.

The deviation risk measures according to what is initially introduced in (Rockafellar, Uryasev and Zabarankin, 2003) is a class of risk measures which generalizes the notion of standard deviation on the space of squarely integrable financial positions $L^{2}(\Omega, \mathcal{F}, \mu)$.

Definition 1.1. A deviation risk measure

$D: L^{2}(\Omega, \mathcal{F}, \mu) \rightarrow[0,+\infty]$ satisfies the following properties:
1) $D(X+c \mathbf{1})=D(X)$ for any $X \in L^{2}$ and for any $c \in \mathbb{R}$, where $\mathbf{1}$ is the constant random variable with $\mathbf{1}(\omega)=1, \omega \in \Omega$.

2) $D(0)=0$ and $D(\lambda X)=\lambda D(X)$ for any $X \in L^{2}$ and for any $\lambda>0$.

3) $D\left(X+X^{\prime}\right) \leq D(X)+D\left(X^{\prime}\right)$ for any $X, X^{\prime} \in L^{2}$.

4) $D(X)>0$ for any $X \in L^{2}$ being non-constant, while $D(X)=0$ if $X$ is constant.

Another class of risk measures which is connected to the deviation measures in (Rockafellar, Uryasev and Zabarankin, 2003 ) is the one of expectation-bounded risk measures, which are defined as follows:

Definition 1.2. An expectation-bounded risk measure $R: L^{2}(\Omega, \mathcal{F}, \mu) \rightarrow(-\infty,+\infty]$ satisfies the following properties:

1) $R(X+c \mathbf{1})=R(X)-c$ for any $X \in L^{2}$ and for any $c \in \mathbb{R}$, where $\mathbf{1}$ is the constant random variable with $\mathbf{1}(\omega)=1, \omega \in \Omega$.

2) $R(0)=0$ and $R(\lambda X)=\lambda R(X)$ for any $X \in L^{2}$ and for any $\lambda>0$.

3) $R\left(X+X^{\prime}\right) \leq R(X)+R\left(X^{\prime}\right)$ for any $X, X^{\prime} \in L^{2}$.

4) $R(X)>\mathbb{E}_{\mu}(-X)$ for any $X \in L^{2}$ being non-constant, while $R(X)=\mathbb{E}_{\mu}(-X)$ if $X$ is constant.

If $R$ is an expectation-bounded risk measure, while $L^{2}$ is partially ordered by the usual partial ordering (denoted by $\geq$ ) and $X \geq Y$ implies $R(Y) \geq R(X)$, then $R$ is coherent in the classical sense of (Artzner, Delbean, Eber, \& Heath, 1999). The seminal survey (Rockafellar, Uryasev and Zabarankin, 2003) contains a lot of themes, such as examples of deviation and expectation-bounded risk measures (see Example 2 in (Rockafellar, Uryasev, \& Zabarankin, 2003), Example 5 in (Rockafellar, Uryasev, \& Zabarankin, 2003)), dual representation (see Theorem 3 of (Rockafellar, Uryasev, \& Zabarankin, 2003)) and portfolio optimization results (see Theorem 4 in (Rockafellar, Uryasev, \& Zabarankin, 2003), Theorem 5 in (Rockafellar, Uryasev, \& Zabarankin, 2003)). Equilibrium in CAPM - like models in which deviation measures are used is studied in (Rockafellar, Uryasev, \& Zabarankin, 2007). Also, results of quantile representation of law-invariant deviation 
measures are proved in (Grechuk, Molyboha, \& Zabarankin, 2009).

The deviation measures were also studied in the published article (Rockafellar, Uryasev, \& Zabarankin, 2006a). Since the properties of a deviation measure are similar to the ones of standard deviation (and this is the explanation for their name), there is also a connection of their properties to those of the class of expectation-bounded risk measures, see for example Theorem 1 in (Rockafellar, Uryasev, \& Zabarankin, 2003). Expectation-bounded measures are a greater class than coherent risk measures (coherent risk measures are mainly studied in (Artzner, Delbean, Eber, \& Heath, 1999), (Delbaen, 2002), (Jaschke \& Küchler, 2001)). Hence we may say that deviation measures is a "bridge" which unifies an "older" and a "newer" aspect on risk functionals. Many of the main results of (Rockafellar, Uryasev, \& Zabarankin, 2003) are transfered to (Rockafellar, Uryasev, \& Zabarankin, 2006a). The major addition of the material contained in (Rockafellar, Uryasev, \& Zabarankin, 2006a) compared to (Rockafellar, Uryasev, \& Zabarankin, 2003) is the Paragraph 4, which is devoted to the error functionals and their relation to deviation measures. Specifically, (Rockafellar, Uryasev, \& Zabarankin, 2006a) contains the above definition of deviation measures (Definition 1 in (Rockafellar, Uryasev, \& Zabarankin, 2006a), while continuity and dual representation results are proved (Proposition 2 of (Rockafellar, Uryasev, \& Zabarankin, 2006a), Theorem 1 of (Rockafellar, Uryasev, \& Zabarankin, 2006a)). The relation between coherent and deviation measures is studied via the class of expectated-bounded risk measures (Theorem 2 of (Rockafellar, Uryasev, \& Zabarankin, 2006a)). The last Theorem indicates that the values of an expectation - bounded measure $R$ on the financial position $X-\mathbb{E}_{\mu}(X) \mathbf{1}, X \in L^{2}$ define a deviation measure and the addition of the term $\mathbb{E}_{\mu}(-X)$ to the value $D(X)$ at any financial position

$X \in L^{2}$, defines an expectation-bounded risk measure $R$. This Theorem is similar to the corresponding generalizations contained in the present article. We extend the content of the Paragraph 4 of (Rockafellar, Uryasev, \& Zabarankin, 2006a) about deviation from error expressions in what we mention in this article about the relation between deviation measures in Banach spaces and seminorms.

The standard one-period problem of minimizing the deviation $D(X)$ is studied in (Rockafellar, Uryasev, \& Zabarankin, 2006b). The random variable $X$ is the linear combination of $\sum_{i=i}^{n} \theta_{i} r_{i}$ in which $r_{i}, i=1,2, \cdots, n$ are the rate of return variables of $n$ assets in $L^{2}$ and $\theta \in \mathbb{R}^{n}$ is a portfolio vector which lies in a polyhedral set of constraints. The problem which arises here is the one of minimizing deviation $D(X)$ subject to the polyhedral constraints. The problem is solved through subgradients which arise from the dual representation of the deviation measures in $L^{2}$ (see Theorem 1 of (Rockafellar, Uryasev, \& Zabarankin, 2006a)). Optimal portfolios are discriminated according to the sum of their coefficients and the financial positions they provide are called master funds. Master funds are either of positive type, or of negative type, or of threshold type, see Theorem 5 in (Rockafellar, Uryasev, \& Zabarankin, 2006b). For all sorts of master funds, CAPM-like relations are deduced, see Definition 3 of (Rockafellar, Uryasev, \& Zabarankin, 2006b). In (Rockafellar, Uryasev, \& Zabarankin, $2006 \mathrm{c}$ ), the random variable $X$ is the convex combination of $\sum_{i=0}^{n} \theta_{i} r_{i}$ in which $r_{i}, i=0,2, \cdots, n$ are the rate of return variables of $n$ assets in $L^{2}$, where $r_{0}$ denotes the risk-free asset rerurn. The problem which arises here is the one of minimizing deviation $D(X)$ subject to a threshold contraint which indicates that the return of the portfolio $\theta$ at the time-period 1 must be more than $r_{0}+\Delta$, where $\Delta$ denotes an amount of money, denoting a risk premium. The existence of some solution to the above problem which is characterized initially either whether the price of the portfolio of the risky assets' price is negative, positive, or equal to 0 , see Theorem 2 of (Rockafellar, Uryasev, \& Zabarankin, 2006c). Master funds are also introduced in this case and efficiency frontiers of expectationdeviation type are studied, related to these master funds, see Paragraph 5 in (Rockafellar, Uryasev, \& Zabarankin, 2006c).

We don't cope with master-funds' portfolio theory in this article. On the contrary, we propose a saddle-point scheme for the minimization of the deviation risk for the choices of an investor which belong to a set $\mathcal{X}$ which is either bounded or unbounded. We consider different min-max Theorems (like the one mentioned in Corollary 3.4 of (Barbu \& Precupanu, 1986), or like the one mentioned in p. 10 of (Delbaen, 2002), in order to prove the existence of solution to the problem of deviation risk minimization for reflexive and non-reflexive spaces. Finally we prove the existence of solution to the general minimization problem with convex contraints' set $\mathcal{X}$ for the wellknown deviation measure $D(X)=E S_{a}(X)+\mathbb{E}_{\mu}(X)$, where $E S_{a}$ denotes the expected shortfall on $L^{1}(\Omega, \mathcal{F}, \mu)$. The portfolio selection problem we study in this article may be compare with the ones contained in (Grechuk, Molyboha, \& Zabarankin, 2011). In the Section 2.2 of (Grechuk, Molyboha, \& Zabarankin, 2011) a cooperative portfolio selection problem is considered which is directly compared to the Markowitz portfolio selection problem in the case of a single investor. The difference is the use of deviation measures.

In the case of the single investor, the Markowitz' type problems - especially the risk minimization over a set of financial positions - is widely studied in our article. We have to mention that throughout the article, we refer to classes of deviation measures defined on Banach spaces whose partial ordering is not the pointwise one in order to indicate the generality of our results. Moreover, as we have also mentioned in (Kountzakis, 2011), the wedge $E_{+}$(which may be actually a cone) by which the partial ordering of $E$ is defined, is a way to interpret "the less and the more", or else when a financial position $x$ is "of greater payoff" than the financial position $y$ whether $x \geq_{E_{f}} y$. Then a rational question is "Who thinks that $x \geq_{E_{+}} y$ "? A possible answer is "All (Some) of the investors of the market do". Let us denote the set of these investors by $I \neq \varnothing$. Every such investor $i \in I$ has her own coherenttype acceptance set $\mathcal{A}_{i} \subseteq E$, which is a wedge of $E$, according to the properties of a coherent risk measure. Namely, the investors may decide to use a deviation measuse $D$ but previously they may have pre-determined by the way of comparing the financial positions according to their initial "risk preferences" indicated by an individual coherent acceptance set $\mathcal{A}_{i}, i \in I$.

Finally, the deviation measures are connected to actuarial science applications and the actuarial approach provided the main motivation about the definition of deviation measures on general Banach spaces. The random variable $X$ of the surplus of an insurance company at a future date $T$ is in general a heavy-tailed one, hence either the positive part $X^{+}=Y_{1}$ or the negative part $X^{-}=Y_{2}$ has the property: For any $\epsilon>0$, $\mathbb{E}_{\mu}\left(\mathrm{e}^{\epsilon Y_{i}}\right)=\infty$ at least for one of $i=1$ or $i=2$. Hence, if for 
example for the maximum $p$ for which $\mathbb{E}_{\mu}\left(|X|^{p}\right)<\infty$, $1 \leq p<\infty$ holds, then $E=L^{p}(\Omega, \mathcal{F}, \mu)$ may be naturally considered as the Banach space of the surplus positions, if the distribution of $X$ is such that leads to this result. Another motivation for this generalization is the actuarial definition of Solvency Capital, as it is mentioned in (Dhaene, Goovaerts, Kaas, Tang, Vanduffel, \& Vyncke, 2003). In this review on risk measures and the notion of solvency the following definition of capital requirement functional for an insurance company is given: if $X$ represents the time- $T$ liabilities of an insurance company and $K(X)$ is the economic capital associated with these liabilites, while $P(X)$ is the value of them calculated either by a quantile method, or by an additional margin method, or by a replicationg portfolio method, then if the risk measure used is $\rho$, the solvency capital for $X$ is equal to $\rho(X)$. The functionals $K, P$ are connected to $\rho$ by the identity $\rho(X)=K(X)+P(X)$ for any $X$ which is a liability variable. If $P=\mathbb{E}_{\mu}$, or else the pricing functional is considered without the margin term, then we may take that $K=\rho-\mathbb{E}_{\mu}$. If $\rho$ is a coherent risk measure, then $K$ is a deviation measure. In these case the insurance company calculates its own Solvency Capital with respect to a generalized risk measure (for example a deviation one), so that it may be acceptable by the regulator. Since the liability variable $X$ is a heavy-tail distributed one, the moments $\mathbb{E}_{\mu}\left(|X|^{p}\right)$ exist till a specific value of $p$. If $p>1$, we may consider $L^{p}(\Omega, \mathcal{F}, \mu)$ or $L^{1}$ to be the model in which we work. This is a motivation for the use of deviation measures on Banach spaces except $L^{2}$.

Another motivation related to financial applications is the class of $L_{G}^{p}$-spaces, which are actually Banach spaces related to G-expectation, see in (Peng, 2007). We may suppose that the variables which denote the value of the portfolios at a certain future date $T$, belong to such spaces, since martingale theory according to the $G$-expectation is related to the $L_{G}^{2}$ space, as (Soner, Touzi, \& Zhang, 2011) indicates. Hence, we may consider the case of definition of deviation measures on this class of Banach spaces. Also, a reference about considering stochastic models of markets under model uncertainty is (Denis \& Martini, 2006). But the definition and the study of deviation measures on $L_{G}^{p}$-spaces should require a separate article.

\section{Deviation Measures on Banach Spaces}

First we remind the definitions of convex and coherent risk measures associated to the Monotonocity property related to the partial ordering defined on $E$ by some wedge $A$ of it.

In the following we refer to the notions of the $(A, e)$-coherent and $(A, e)$-convex risk measures (where $E$ is partially ordered by the partial ordering relation induced by the wedge $A$ of it), whose definitions are the following:

Definition 2.1. A function $\rho: E \rightarrow(-\infty,+\infty]$ which satisfies the properties

1) $\rho(x+a e)=\rho(x)-a \quad(e-$ Translation Invariance $)$.

2) $\rho(\lambda x+(1-\lambda) x) \leq \lambda \rho(x)+(1-\lambda) \rho(y)$ for any

$\lambda \in[0,1]$ (Convexity).

3) $y \geq_{A} x$ implies $\rho(y) \leq \rho(x)$ (A-Monotonicity). where $x, y \in E$ is called $(A, e)$-convex risk measure.

Definition 2.2. A function $\rho: E \rightarrow(-\infty,+\infty]$ is a $(A, e)$ coherent risk measure if it is an $(A, e)$-convex risk measure and it satisfies the following property: $\rho(\lambda x)=\lambda \rho(x)$ for any $x \in E$ and any $\lambda \in \mathbb{R}_{+}$(Positive Homogeneity).

In both of these definitions, we suppose that $\rho \neq I_{\{+\infty\}}$, being the characteristic function of the value $+\infty$.

Let $E$ be a Banach space, being partially ordered by a closed cone $P$ of $E$. Also consider a non-trivial, closed subspace $K$ of $E$. Suppose that this cone has a base $B_{\ell}$ defined by a continuous linear functional $\ell$ of $E$, namely that $B_{\ell}=\{x \in P \mid \ell(x)=1\}$.

Definition 2.3. A $K$-deviation risk measure

$D: E \rightarrow[0,+\infty]$ satisfies the following properties:

1) $D(x+c t)=D(x)$ for any $x \in E$ and for any $c \in \mathbb{R}$ and any $t \in K \cap B_{\ell}$.

2) $D(0)=0$ and $D(\lambda x)=\lambda D(x)$ for any $x \in E$ and for any $\lambda>0$.

3) $D\left(x+x^{\prime}\right) \leq D(x)+D\left(x^{\prime}\right)$ for any $x, x^{\prime} \in E$.

4) $D(x)>0$ for any $x \in E \backslash K$, while $D(x)=0$ if $x \in K$.

The definition of the $K$-expectation-bounded risk measures is the following:

Definition 2.4. A $K$-expectation-bounded risk measure $R: E \rightarrow(-\infty,+\infty]$ satisfies the following properties:

1) $R(x+c e)=R(x)-c$ for any $x \in E$ and for any $c \in \mathbb{R}$ and any $e \in K \cap B_{\ell}$.

2) $R(0)=0$ and $R(\lambda x)=\lambda R(x)$ for any $x \in E$ and for any $\lambda>0$.

3) $R\left(x+x^{\prime}\right) \leq R(x)+R\left(x^{\prime}\right)$ for any $x, x^{\prime} \in E$.

4) $R(x)>\ell(-x)$ for any $x \in E \backslash K$, while $R(x)=\ell(-x)$ if $x \in K$.

If $R$ is a $K$-expectation-bounded risk measure, while $E$ is partially ordered by the usual partial ordering induced by $P$ (denoted by $\geq_{p}$ ) and $x \geq_{p} y$ implies $R(y) \geq R(x)$, then $R$ is $(P, e)$-coherent.

Proposition 2.5. If $D$ is a $K$-deviation measure on $E$, the functional $R_{D}: E \rightarrow(-\infty,+\infty]$, where

$$
R_{D}(x)=D(x)-\ell(x), x \in E,
$$

is a $K$-expectation bounded risk measure.

Proof. It suffices to prove that $R_{D}$ satisfies the properties of a $K$-expectation bounded risk measure on the partially ordered Banach space $E$.

1) $R_{D}(x+c t)=R_{D}(x)-c$ for any $x \in E$ and for any $c \in \mathbb{R}, t \in K \cap B_{\ell}$. This property holds due to the definition of $R_{D}$ and the equivalent property of $D$, which is a $K$-deviation measure, namely

$$
\begin{aligned}
R_{D}(x+c t) & =D(x+c t)-\ell(x+c t) \\
& =D(x)-\ell(x)-c \ell(t) \\
& =R_{D}(x)-c .
\end{aligned}
$$

2) $R_{D}(0)=0$ and $R_{D}(\lambda x)=\lambda R_{D}(x)$ for any $x \in E$ and for any $\lambda>0$. This property also holds due to the definition of $R_{D}$ and the equivalent property of $D$ as a deviation measure, namely $R_{D}(0)=D(0)-\ell(0)=0$ and

$$
R_{D}(\lambda x)=D(\lambda x)-\ell(\lambda x)=\lambda D(x)-\lambda \ell(x)=\lambda R_{D}(x),
$$

if $\lambda>0$ and for any $x \in E$.

3) $R_{D}\left(x+x^{\prime}\right) \leq R_{D}(x)+R_{D}\left(x^{\prime}\right)$ for any $x, x^{\prime} \in E$. By the same way, we have that

$$
\begin{aligned}
R_{D}\left(x+x^{\prime}\right) & =D\left(x+x^{\prime}\right)-\ell\left(x+x^{\prime}\right) \\
& \leq D(x)+D\left(x^{\prime}\right)-\ell(x)-\ell\left(x^{\prime}\right)
\end{aligned}
$$




\section{E. KOUNTZAKIS}

which implies $R_{D}\left(x+x^{\prime}\right) \leq R_{D}(x)+R_{D}\left(x^{\prime}\right)$ for any $x, x^{\prime} \in E$.

4) $R_{D}(x)>\ell(-x)$ for any $x \in E \backslash K$, while $R_{D}(x)=\ell(-x)$ if $x \in K$. Since $D(x)>0$ if $x \in E \backslash K$ then $R_{D}(x)=D(x)-\ell(x)>-\ell(x)=\ell(-x)$ if $x \in E \backslash K$. If $x \in K$, then $D(x)=0$, hence

$R_{D}(x)=D(x)-\ell(x)=-\ell(x)=\ell(-x)$.

Proposition 2.6. If $R$ is a $K$-expectation bounded risk measure on $E$, the functional $D_{R}: E \rightarrow[0,+\infty]$, where

$$
D_{R}(x)=R(x)+\ell(x),
$$

is a $K$-deviation risk measure.

Proof. It suffices to prove that $D_{R}$ satisfies the properties of a $K$-deviation risk measure.

1) $D_{R}(x+c t)=D_{R}(x)$ for any $x \in E$ and for any $c \in \mathbb{R}$, where $t \in K \cap B_{\ell}$. From the definition of $D_{R}$ and the equivalent translation invariant property of the risk measure $R$, we have that

$$
\begin{aligned}
D_{R}(x+c t) & =R(x+c t)+\ell(x+c t) \\
& =R(x)-c+\ell(x)+c \ell(t) \\
& =R(x)+\ell(x)=D_{R}(x) .
\end{aligned}
$$

2) $D_{R}(0)=0$ and $D_{R}(\lambda x)=\lambda D_{R}(x)$ for for any $x \in E$ and for any $\lambda>0$. From the definition of $D_{R}$ and the equivalent property of the $K$-expectation bounded risk measure $R$, we have $D_{R}(0)=R(0)+\ell(0)=0$ and

$$
\begin{aligned}
D_{R}(\lambda x) & =R(\lambda x)+\ell(\lambda x) \\
& =\lambda R(x)+\lambda \ell(x)=\lambda D_{R}(x),
\end{aligned}
$$

for every $\lambda>0$ and $x \in E$.

3) $D_{R}\left(x+x^{\prime}\right) \leq D_{R}(x)+D_{R}\left(x^{\prime}\right)$ for any $x, x^{\prime} \in E$. By the same way we have that

$$
\begin{aligned}
D_{R}\left(x+x^{\prime}\right) & =R\left(x+x^{\prime}\right)+\ell\left(x+x^{\prime}\right) \\
& \leq R(x)+R\left(x^{\prime}\right)+\ell(x)+\ell\left(x^{\prime}\right) \\
& =D_{R}(x)+D_{R}\left(x^{\prime}\right) .
\end{aligned}
$$

4) $D_{R}(x)>0$ for any $x \in E \backslash K$, while $D_{R}(x)=0$ if $x \in K$. Since $R(x)>\ell(-x)$ if $x \in E \backslash K$, then $D_{R}(x)=R(x)-\ell(x)>0$ in this case. Also, $R(x)=\ell(-x)$ if $x \in K$, which implies that $D_{R}(x)=R(x)-\ell(x)=0$ in this case, too.

Let us see some examples, classes of deviation risk measures which are defined on partially ordered Banach spaces by using coherent risk measures, which are actually expectation-bounded risk measures.

Corollary 2.7. Suppose that $\mathcal{P} \subseteq P^{0}$ such that the functionals of $\mathcal{P}$ are strictly positive functionals of $P$ and $K$ is a closed, non-trivial subspace of $E$ such that

$\pi(-x)-\ell(-x)=0$ for any $x \in K$ and any $\pi \in \mathcal{P}$. On the other hand, for any $x \in E \backslash K$ there is $a \pi_{x} \in \mathcal{P}$ such that $\pi_{x}(-x)>\ell(-x)$, then the functional $D_{R_{p}}: E \rightarrow \mathbb{R}$, where

$$
D_{R_{\mathcal{P}}}(x)=\sup \{\pi(-x) \mid \pi \in \mathcal{P}\}+\ell(x),
$$

is a $K$-deviation risk measure.

Proof. We have to prove that $D_{R_{\mathcal{P}}}$ satisfies the properties of a $K$-deviation risk measure.

1) $D_{R_{p}}(x+c t)=D_{R_{P}}(x)$ for any $x \in E$ and for any $c \in \mathbb{R}$, where $t \in K \cap B_{\ell}$. From the definition of $D_{R_{p}}$ and the definition of the risk measure $R$, we have that

$$
\begin{aligned}
D_{R_{\mathcal{P}}}(x+c t) & =R_{\mathcal{P}}(x+c t)+\ell(x+c t) \\
& =\sup \{\pi(x+c t) \mid \pi \in \mathcal{P}\}+\ell(x)+c \ell(t) \\
& =\sup \{\pi(-x)+c \pi(-t) \mid \pi \in \mathcal{P}\}+\ell(x)+c \ell(t) \\
& =\sup \{\pi(-x)-c \mid \pi \in \mathcal{P}\}+\ell(x)+c \\
& =R_{\mathcal{P}}(x)-c+\ell(x)+c \\
& =R_{\mathcal{P}}(x)+\ell(x)=D_{R_{\mathcal{P}}}(x) .
\end{aligned}
$$

2) $D_{R_{p}}(0)=0$ and $D_{R_{p}}(\lambda x)=\lambda D_{R_{p}}(x)$ for any $x \in E$ and for any $\lambda>0$. From the definition of $D_{R_{P}}$, we have $D_{R_{\mathcal{P}}}(0)=R_{\mathcal{P}}(0)+\ell(0)=\sup \{\pi(0) \mid \pi \in \mathcal{P}\}+\ell(0) \stackrel{R_{\mathcal{P}}}{=} 0$ and

$$
\begin{aligned}
D_{R_{\mathcal{P}}}(\lambda x) & =R_{\mathcal{P}}(\lambda x)+\ell(\lambda x)=\sup \{\pi(-\lambda x) \mid \pi \in \mathcal{P}\}+\ell(\lambda x) \\
& =\lambda R_{\mathcal{P}}(x)+\lambda \ell(x)=\lambda D_{R_{\mathcal{P}}}(x),
\end{aligned}
$$

for every $\lambda>0$ and $x \in E$.

3) $D_{R_{p}}\left(x+x^{\prime}\right) \leq D_{R_{p}}(x)+D_{R_{p}}\left(x^{\prime}\right)$ for any $x, x^{\prime} \in E$. We have that

$$
\begin{aligned}
D_{R_{\mathcal{P}}}\left(x+x^{\prime}\right) & =R_{\mathcal{P}}\left(x+x^{\prime}\right)+\ell\left(x+x^{\prime}\right) \\
& \leq R_{\mathcal{P}}(x)+\ell(x)+R_{\mathcal{P}}\left(x^{\prime}\right)+\ell\left(x^{\prime}\right) \\
& =D_{R_{\mathcal{P}}}(x)+D_{R_{\mathcal{P}}}\left(x^{\prime}\right) .
\end{aligned}
$$

Also, we remind that

$$
\begin{aligned}
R_{\mathcal{P}}\left(x+x^{\prime}\right) & =\sup \left\{\pi\left(-x-x^{\prime}\right) \mid \pi \in \mathcal{P}\right\} \\
& =\sup \left\{\pi(-x)+\pi\left(-x^{\prime}\right) \mid \pi \in \mathcal{P}\right\} \\
& \leq \sup \{\pi(-x) \mid \pi \in \mathcal{P}\}+\sup \left\{\pi\left(-x^{\prime}\right) \mid \pi \in \mathcal{P}\right\} \\
& =R_{\mathcal{P}}(x)+R_{\mathcal{P}}\left(x^{\prime}\right) .
\end{aligned}
$$

4) If $\pi(-x)=\ell(-x)$ for any $x \in K$ and any $\pi \in \mathcal{P}$, then $\sup \{\pi(-x) \mid \pi \in \mathcal{P}\}=\ell(-x)$, which implies that

$D_{R_{\mathcal{P}}}(x)=\sup \{\pi(-x) \mid \pi \in \mathcal{P}\}+\ell(x)=0$. On the other hand, if $x \in E \backslash K$, then there is some $\pi_{x} \in \mathcal{P}$ such that $\pi_{x}(-x)-\ell(-x) \neq 0$. If $\pi_{x}(-x)>\ell(-x)$, this implies that $\sup \{\pi(-x) \mid \pi \in \mathcal{P}\} \geq \pi_{x}(-x)>\ell(-x)$. Hence,

$D_{R_{P}}(x)=\sup \{\pi(-x) \mid \pi \in \mathcal{P}\}+\ell(x)>0$. If $\pi_{x}(-x)<\ell(-x)$, we may repeat the same argument for $-x \in E \backslash K$.

Corollary 2.8. Suppose that $E$ is reflexive, $K$ is a nontrivial, closed subspace of $E, P$ is a closed cone of $E$ with int $P \neq \varnothing, \quad e \in K \bigcap$ int $P$, while $\rho: E \rightarrow(-\infty,+\infty)$ is a $(P, e)$-coherent risk measure. We also suppose that the acceptance set $\mathcal{A}_{\rho}$ is $\sigma\left(E, E^{*}\right)$-closed. Moreover, suppose that $\pi(-x)-\ell(-x)=0$ for any $x \in K$ and any $\pi \in B$. On the other hand, for any $x \in E \backslash K$ there is $a \pi_{x} \in B$ such that $\pi_{x}(-x)>\ell(-x)$, where $B=B_{e} \cap \mathcal{A}_{\rho}^{0}$ and $B_{e}$ is the base defined by $e$ on $P^{0}$. Then $D_{\rho}: E \rightarrow[0,+\infty)$ which is defined by

$$
D_{\rho}(x)=\rho(x)+\ell(x),
$$

is a $K$-deviation risk measure.

Proof. Since $\rho$ is a $(P, e)$-coherent risk measure (see Theorem 3.1 of (Konstantinides \& Kountzakis, 2011)), it is also a $K$-expectation bounded risk measure, since $e \in K$. Hence, 


\section{E. KOUNTZAKIS}

by the Proposition 2.6, $D_{\rho}$ is a $K$-deviation risk measure.

Corollary 2.9. Suppose that $E$ is non-reflexive, $K$ is a non-trivial, closed subspace of $E, P$ is a closed cone of $E^{*}$ with $\operatorname{int} P_{0} \neq \varnothing$ and $e \in K \bigcap$ int $P$, while $\rho: E \rightarrow(-\infty,+\infty]$ is a $\left(P_{0}, e\right)$-coherent risk measure whose acceptance set $\mathcal{A}_{\rho}$ is $\sigma\left(E, E^{*}\right)$-closed. Moreover, suppose that

$\pi(-x)-\ell(-x)=0$ for any $x \in K$ and any $\pi \in B$. On the other hand, for any $x \in E \backslash K$ there is $a \pi_{x} \in B$ such that $\pi_{x}(-x)>\ell(-x)$, where $B=B_{e} \cap \mathcal{A}_{\rho}^{0}$ and $B_{e}$ is the base defined by $e$ on $P$. Then $D_{\rho}: E \rightarrow(0,+\infty)$ which is defined by

$$
D_{\rho}(x)=\rho(x)+\ell(x),
$$

is a $K$-deviation risk measure.

Proof. Since $\rho$ is a $\left(P_{0}, e\right)$-coherent risk measure (see Theorem 3.5 of (Kountzakis, 2011)), it is also a $K$-expectation bounded risk measure, since $e \in K$. Hence, by the Proposition $2.6 D_{\rho}$ is a $K$-deviation risk measure.

Since in Definition 1 of (Rockafellar, Uryasev and Zabarankin, 2003) the deviation measures are defined on $L^{2}$ spaces, we may state and prove similar Corollaries for the usual (component-wise partial ordering) of $L^{p}$ spaces with $1 \leq p<\infty$.

We rely on the unified dual representation Theorem 2.9 of (Kaina \& Rüschendorf, 2009) in order to state the following:

Corollary 2.10. If $\rho: L^{p} \rightarrow(-\infty,+\infty)$ is a $\left(L_{+}^{p}, \mathbf{1}\right)$-coherent risk measure, where $1 \leq p<\infty$, then $D_{\rho}$ where $D_{\rho}: L^{p} \rightarrow[0,+\infty)$ with $D_{\rho}(x)=\rho(x)+\mathbb{E}_{\mu}(x)$ is a $K-$ deviation risk measure with $K=\{x=\lambda \mathbf{1} \mid \lambda \in \mathbb{R}\}$.

Proof. Since by Theorem 2.9 of (Kaina \& Rüschendorf, 2009), a finite-valued, coherent risk measure $\rho$ is representable as $\rho(x)=\max \left\{\mathbb{E}_{Q}(-x) \mid Q \in \mathcal{Q}\right\}$, where $\mathcal{Q} \subseteq \mathcal{Q}_{p}$ and

$$
\mathcal{Q}_{p}=\left\{Q \in M_{1} \mid \frac{\mathrm{d} Q}{\mathrm{~d} \mu} \in L^{q}\right\}, 1 \leq p<\infty,
$$

where $q$ is such that $\frac{1}{p}+\frac{1}{q}=1$, while $M_{1}$ denotes the set of $\mu$-continuous probability measures on the measurable space $(\Omega, \mathcal{F})$. Let us denote by $\pi_{Q}$ the functional $\pi_{Q} \in B_{1}$ lying in the base defined on $L_{+}^{q}$ by $\mathbf{1}$. Here we refer to the case where $E=L^{p}, 1 \leq p<\infty . \pi_{Q}=\frac{\mathrm{d} Q}{\mathrm{~d} \mu}$ is actually the Radon-

Nikodym derivative of $Q$ with respect to $\mu$.

It suffices to prove that $D_{\rho}$ satisfies the properties of a $K$-deviation risk measure.

1) $D_{\rho}(x+c \mathbf{1})=D_{\rho}(x)$ for any $x \in L^{p}$ and for any $c \in \mathbb{R}$, where $c \in \mathbb{R}$. From the definition of $D_{\rho}$ and the Translation Invariance of the risk measure $\rho$, we have that

$$
\begin{aligned}
D_{\rho}(x+c \mathbf{1}) & =\rho(x+c \mathbf{1})+\mathbb{E}_{\mu}(x+c \mathbf{1})=\rho(x)-c+\mathbb{E}_{\mu}(x)+c \\
& =\rho(x)+\mathbb{E}_{\mu}(x)=D_{\rho}(x) .
\end{aligned}
$$

2) $D_{\rho}(0)=0$ and $D_{\rho}(\lambda x)=\lambda D_{\rho}(x)$ for any $x \in L^{p}$,

$1 \leq p<\infty$ and for any $\lambda>0$. From the definition of $D_{\rho}$ and the Positive Homogeneity of $\rho$, we have

$$
\begin{aligned}
& D_{\rho}(0)=\rho(0)+\mathbb{E}_{\mu}(0)=0 \text { and } \\
& \begin{aligned}
D_{\mu}(\lambda x) & =\rho(\lambda x)+\mathbb{E}_{\mu}(\lambda x)=\lambda \rho(x)+\lambda \mathbb{E}_{\mu}(x) \\
& =\lambda D_{\rho}(x),
\end{aligned}
\end{aligned}
$$

for every $\lambda>0$ and $x \in L^{p}, 1 \leq p<\infty$.

3) $D_{\rho}\left(x+x^{\prime}\right) \leq D_{\rho}(x)+D_{\rho}\left(x^{\prime}\right)$ for any $x, x^{\prime} \in L^{p}$, $1 \leq p<\infty$. By the same way we have that

$$
\begin{aligned}
D_{\rho}\left(x+x^{\prime}\right) & =\rho\left(x+x^{\prime}\right)+\mathbb{E}_{\mu}\left(x+x^{\prime}\right) \\
& \leq \rho(x)+\rho\left(x^{\prime}\right)+\mathbb{E}_{\mu}(x)+\mathbb{E}_{\mu}\left(x^{\prime}\right) \\
& =D_{\rho}(x)+D_{\rho}\left(x^{\prime}\right),
\end{aligned}
$$

since $\rho$ is Subadditive.

4) If $x \in K$, then there is some $c \in \mathbb{R}$ such that $x=c \mathbf{1}$, $\mu$-a.e. Then $\pi_{Q}(-x)=-c$ for any $Q \in \mathcal{Q}$ such that $\rho(x)=\max \left\{\mathbb{E}_{Q}(-x) \mid Q \in \mathcal{Q}\right\}$ for any $x \in E$. We also remind that $\pi_{Q}(x)=\mathbb{E}_{Q}(x)$ for any $x \in E$. Moreover $\mathbb{E}_{\mu}(-x)=-c$ for any $x \in K$, which indicates that $\pi_{Q}(-x)=\mathbb{E}_{\mu}(-x)$ for any $x \in K$ and any $Q \in \mathcal{Q}$ such that $\rho(x)=\max \left\{\mathbb{E}_{Q}(-x) \mid Q \in \mathcal{Q}\right\}$. If $x \in E \backslash K$, then there is some $Q_{x} \in \mathcal{Q}$ such that $\mathbb{E}_{Q_{x}}(-x) \neq \mathbb{E}_{\mu}(-x)$. If $\mathbb{E}_{Q_{x}}(-x)>\mathbb{E}_{\mu}(-x)$, then $\max \left\{\mathbb{E}_{Q}(-x) \mid Q \in \mathcal{Q}\right\} \geq \mathbb{E}_{Q_{x}}(-x)>\mathbb{E}_{\mu}(-x)$, which implies that $\rho(x)>\mathbb{E}_{\mu}(-x)$ and this implies that $D_{\rho}(x)=\rho(x)+\mathbb{E}_{\mu}(x)>0$. If $\mathbb{E}_{Q_{x}}(-x)<\mathbb{E}_{\mu}(-x)$, then we may repeat the same argument for $-x \in E \backslash K$.

Another example of $K$-deviation measures arises if we depart from the component-wise partial ordering of $L^{p}$-spaces.

Example 2.11. If $E=L^{1}(\Omega, \mathcal{F}, \mu), e=1$ and $L^{\infty}(\Omega, \mathcal{F}, \mu)$ is partially ordered by the cone

$P=\left\{y \in L^{\infty}(\Omega, \mathcal{F}, \mu) \mid \int_{\Omega} y(\omega) \mathrm{d} \mu(\omega) \geq \frac{1}{2}\|y\|_{\infty}\right\}$, then

$\mathbf{1} \in \operatorname{int}\left(P_{0}\right)$ and we may suppose that $L^{1}(\Omega, \mathcal{F}, \mu)$ is partially ordered by the wedge $P_{0} \neq L_{+}^{1}$. Then every $\left(P_{0}, \mathbf{1}\right)$-coherent risk measure $\rho: L^{1}(\Omega, \mathcal{F}, \mu) \rightarrow(-\infty,+\infty]$ whose acceptance set $\mathcal{A}_{\rho}$ is weakly closed, is represented in the way that Theorem 3.5 of (Kountzakis, 2011) indicates:

$$
\rho(x)=\sup \left\{\pi(-x) \mid \pi \in B_{1}\right\} .
$$

We have to verify that $D: L^{1}(\Omega, \mathcal{F}, \mu) \rightarrow[0,+\infty]$ with $D(x)=\rho(x)+\mathbb{E}_{\mu}(x)$ for any $x \in L^{1}(\Omega, \mathcal{F}, \mu)$ is a

$K$-deviation measure, if $K$ is also the subspace of the constant random variables.

It suffices to prove that $D$ satisfies the properties of a $K$-deviation risk measure.

1) $D(x+c \mathbf{1})=D_{\rho}(x)$ for any $x \in L^{1}$ and for any $c \in \mathbb{R}$, where $c \in \mathbb{R}$. From the definition of $D$ and the Translation Invariance of the risk measure $\rho$, we have that

$$
\begin{aligned}
D(x+c 1) & =\rho(x+c 1)+\mathbb{E}_{\mu}(x+c 1)=\rho(x)-c+\mathbb{E}_{\mu}(x)+c \\
& =\rho(x)+\mathbb{E}_{\mu}(x)=D_{\rho}(x) .
\end{aligned}
$$

2) $D(0)=0$ and $D(\lambda x)=\lambda D(x)$ for any $x \in L^{1}$ and for any $\lambda>0$. From the definition of $D$ and the Positive Homogeneity of $\rho$, we have $D_{\rho}(0)=\rho(0)+\mathbb{E}_{\mu}(0)=0$ and

$$
D(\lambda x)=\rho(\lambda x)+\mathbb{E}_{\mu}(\lambda x)=\lambda \rho(x)+\lambda \mathbb{E}_{\mu}(x)=\lambda D(x),
$$

for every $\lambda>0$ and $x \in L^{1}$.

3) $D\left(x+x^{\prime}\right) \leq D(x)+D\left(x^{\prime}\right)$ for any $x, x^{\prime} \in L^{1}$. By the same way we have that

$$
\begin{aligned}
D\left(x+x^{\prime}\right) & =\rho\left(x+x^{\prime}\right)+\mathbb{E}_{\mu}\left(x+x^{\prime}\right) \\
& \leq \rho(x)+\rho\left(x^{\prime}\right)+\mathbb{E}_{\mu}(x)+\mathbb{E}_{\mu}\left(x^{\prime}\right)=D(x)+D\left(x^{\prime}\right),
\end{aligned}
$$




\section{E. KOUNTZAKIS}

since $\rho$ is Subadditive.

4) $\rho(x)=\mathbb{E}_{\mu}(-x)$ if $x \in K$, since $\rho(c \mathbf{1})=-c$,

$\mathbb{E}_{\mu}(c \mathbf{1})=c$ and $x=c \mathbf{1}$ if $x \in K$, which implies that

$D(x)=\rho(x)+\mathbb{E}_{\mu}(x)=0$ in this case. Also, we may notice that if $x \notin K$, then there is a co-set $y_{0}+K$ with $y_{0} \notin K$, such that $x \in y_{0}+K$. Then $x=y_{0}+k_{0} \mathbf{1}$ for some $k_{0} \in \mathbb{R}$. But from the first property $D(x)=D\left(y_{0}\right)$, hence it suffices to prove that $D\left(y_{0}\right)>0$ in any equivalent case. Notice that if $x \in K$, then $\pi(-x)=\mathbb{E}_{\mu}(-x)$ for any $\pi \in B$. This implies that if $y_{0} \notin K$, then there is some $\pi_{0} \in B$ such that $\pi_{0}\left(-y_{0}\right) \neq \mathbb{E}_{\mu}\left(-y_{0}\right)$. If $\pi_{0}\left(-y_{0}\right)>\mathbb{E}_{\mu}\left(-y_{0}\right)$, then $\sup \left\{\pi\left(-y_{0}\right) \mid \pi \in B\right\} \geq \pi_{0}\left(-y_{0}\right)>\mathbb{E}_{\mu}\left(-y_{0}\right)$, which implies what we wanted to prove. If $\pi_{0}\left(-y_{0}\right)<\mathbb{E}_{\mu}\left(-y_{0}\right)$, then apply again the previous argument for $-y_{0} \notin K$.

Since the value of a risk measure at any financial position has both the financial and the actuarial interpretation of the premium, the term $\ell(x)$ corresponds to a standard term of the risk premium, which is related to the geometry of the acceptance set. When the acceptance set is the positive cone $L_{+}^{2}$ of the space of the square-integrable risks, then this standard term is the mean value $\mathbb{E}_{\mu}(x)$, since $\ell=1$ in this case. The last case is the usual attitude towards risk, under which a non-risky position is a position whose outcomes are positive $\mu$-almost everywhere in $\Omega$.

The subspace $K$ mentioned in the Definition 2.3 above, may be considered to be a subspace of non-risky assets. For this reason, the addition of such an asset does not affect the premium calculation, according to the first property of the $K$ deviation measures. However, the whole theory of $K$-deviation measures can be developed without reference to the partial ordering.

Consider a proper subspace of assets (which are considered to be the non-risky ones), denoted by $K$.

Definition 2.12. A $K$-deviation risk measure

$D: E \rightarrow[0,+\infty]$ satisfies the following properties:

1) $D(x+k)=D(x)$ for any $x \in E$ and for any $c \in \mathbb{R}$, where $k \in K$, where $K$ is a closed subspace of $E$.

2) $D(0)=0$ and $D(\lambda x)=\lambda D(x)$ for for any $x \in E$ and for any $\lambda>0$.

3) $D\left(x+x^{\prime}\right) \leq D(x)+D\left(x^{\prime}\right)$ for any $x, x^{\prime} \in E$.

4) $D(x)>0$ for any $x \in E \backslash K$, while $D(x)=0$ if $x \in K$.

The definition of the $K$-expectation-bounded risk measures is the following:

Definition 2.13. A $K$ expectation-bounded risk measure $R: E \rightarrow(-\infty,+\infty]$ satisfies the following properties:

1) $R(x+c k)=R(x)-c$ for any $x \in E$ and for any $c \in \mathbb{R}, \quad k \in K$

2) $R(0)=0$ and $R(\lambda x)=\lambda R(x)$ for any $x \in K$ and for any $\lambda>0$.

3) $R\left(x+x^{\prime}\right) \leq R(x)+R\left(x^{\prime}\right)$ for any $x, x^{\prime} \in E$.

4) $R(x)>\ell(-x)$ for any $x \in E \backslash K$, while

$R(x)=\ell(-x)$ if $x \in K$.

Proposition 2.14. A $K$-deviation measure $D$ defines $a$ seminorm $g_{D}$ on $E$.

Proof. The conclusion is immediate, since by property (ii) $D$ is positively homogeneous and by property (iii) $D$ is subadditive, hence it is sublinear, according to Definition 5.32 of (Aliprantis and Border, 1999). This implies by Lemma 5.33 of (Aliprantis and Border, 1999) that the function $g_{D}: E \rightarrow \mathbb{R}$ defined by $g_{D}(x)=\max \{D(x), D(-x)\}$ is a seminorm on $E$. Actually, $g_{D}(x+y)=\max \{D(x+y), D(-x-y)\}$ and since $D$ is Subadditive, we have that

$D(x+y) \leq D(x)+D(y), D(-x-y) \leq D(-x)+D(-y)$. Hence

$$
\begin{aligned}
g_{D}(x+y) & =\max \{D(x+y), D(-x-y)\} \\
& \leq \max \{D(x), D(-x)\}+\max \{D(y), D(-y)\} \\
& =g_{D}(x)+g_{D}(y),
\end{aligned}
$$

by the properties of maximum of real numbers. Hence $g_{D}$ is Subadditive. Also, by Homogeneity Property of $D$, we have that

$$
\begin{aligned}
g_{D}(\lambda x) & =\max \{D(\lambda x), D(-\lambda x)\} \\
& =\max \{\lambda D(x), \lambda D(-x)\}
\end{aligned}
$$

if $\lambda>0$ and also by well-known properties of maximum of real numbers,

$$
g_{D}(\lambda x)=\lambda \max \{D(x), D(-x)\}=\lambda g_{D}(x) .
$$

If $\lambda<0$, then

$$
\begin{aligned}
g_{D}(\lambda x) & =\max \{D(\lambda x), D(-\lambda x)\} \\
& =\max \{(-\lambda) D(-x),(-\lambda) D(x)\}
\end{aligned}
$$

which is equal to $(-\lambda) g_{D}(x)$ for the same reason. Also, if $\lambda=0$ then $g_{D}(\lambda X)=\max \{D(0), D(0)\}=0=\lambda g_{D}(x)$ for any $x \in E$. Finally, $g_{D}(\lambda x)=|\lambda| g_{D}(x)$ for any $x \in E$ and any $\lambda \in \mathbb{R}$.

The same proof may be repeated for $K$-deviation measures defined on partially ordered spaces.

Corollary 2.15. A $K$-deviation measure $D$ defines a seminorm $g_{D}$ on $E$.

Also, by the above Proposition, another Corollary arises for the deviation measures which were initially defined on $L^{2}(\Omega, \mathcal{F}, \mu)$.

Corollary 2.16. A deviation measure $D$ defines a seminorm $g_{D}$ on $L^{2}(\Omega, \mathcal{F}, \mu)$.

Another result concerning seminorms is the following.

Proposition 2.17. A seminorm $p$ defined on $E$ such that $K=\{x \in E \mid p(x)=0\}$ is a $K$-deviation measure.

Proof. It suffices to prove that $p$ satisfies the properties of a $K$-deviation measure.

1) $p(x+k)=p(x)$ for any $x \in E$ and for any $k \in K$. This holds due to the subadditivity property of the seminorm $p$ according to which,

$$
p(x+k) \leq p(x)+p(k)
$$

and

$$
p(x) \leq p(x+k)+p(-k)
$$

while $p(k)=0, p(-k)=0$ for any $k \in K, x \in E$. Hence the equality $p(x+k)=p(x)$ is true.

2) $p(0)=0$ and $p(\lambda x)=\lambda p(x)$ for any $x \in E$ and for any $\lambda>0$, since $p(\lambda x)=|\lambda| p(x)$ for any $\lambda \in \mathbb{R}$ and any $x \in E$.

3) $p\left(x+x^{\prime}\right) \leq p(x)+p\left(x^{\prime}\right)$ for any $x, x^{\prime} \in E$, from the subadditivity of the seminorm $p$. 
4) $p(x)>0$ for any $x \in E \backslash K$, while $p(x)=0$ if $x \in K$. Consider the co-sets $y+K, y \in E$. Every $x \in E$ belongs to some of these co-sets. If $x \in K$, then it belongs to the co-set $0+K=K$, hence $p(x)=0$ holds. If $x \in E \backslash K$, then it belongs to some co-set of the form $y_{0}+K$, where $y_{0} \notin K$. Then $x=y_{0}+k_{0}$ for some $k_{0} \in K$. This implies $p(x)=p\left(y_{0}+k_{0}\right)=p\left(y_{0}\right)>0$.

Again, by the above Proposition, we obtain another Corollary for the deviation measures which were initially defined on $L^{2}(\Omega, \mathcal{F}, \mu)$.

Corollary 2.18. A seminorm $p$ defined on $E$ such that $K=\{x \in E \mid p(x)=0\}=\{x \in E \mid x=c \cdot \mathbf{1}, c \in \mathbb{R}\}$, is actually a $K$-deviation measure.

The same proof may be repeated for $K$-deviation measures defined on partially ordered spaces in the sense we defined them before, hence we obtain the following

Corollary 2.19. A seminorm $p$ defined on $E$ such that $K=\{x \in E \mid p(x)=0\}=\{x \in E \mid x=c \cdot e, c \in \mathbb{R}\}$, where $e \in B_{\ell}$, is actually a $K$-deviation measure.

Proof. In both of cases of $L^{2}$ and the case of the above Corollary, we repeat the proof of Proposition 2.17 In the case of $L^{2}$ we replace $k \in K$ by $c \cdot \mathbf{1}$, while in the case of an ordered Banach space we replace $k \in K$ by $c \cdot e, e \in B_{\ell}$, where $c \in \mathbb{R}$.

Example 2.20. Consider a set of continuous linear functionals $\left\{f_{i} \mid i \in I\right\}$ of $E$, where $\left\|f_{i}\right\|=1$ in $E^{*}$ and $I \neq \varnothing$. Also, suppose that $K=\cap_{i \in I}$ kerf, where $K \neq\{0\}_{i}$. Then the functional $p_{I}: E \rightarrow \mathbb{R}_{+}$, where $p_{I}(x)=\sup \left\{\left|f_{i}(x)\right| \mid i \in I\right\}$ is a seminorm on $E$ with $\left\{x \in E \mid p_{I}(x)=0\right\}=K$. Note that $p_{I}(x)$ is a real number for any $x \in E$ since

$\left|p_{I}(x)\right| \leq \sup \left\{\left\|f_{i}\right\| \cdot\|x\| i \in I\right\} \leq\|x\|$. For the subadditivity of $p_{I}$ we have that

$$
\begin{aligned}
p_{i}(x+y) & =\sup \left\{\mid f_{i}(x+y) \| i \in I\right\} \\
& \leq \sup \left\{\left|f_{i}(x)\right|+\mid f_{i}(y) \| i \in I\right\} \\
& \leq \sup \left\{\mid f_{i}(x) \| i \in I\right\}+\sup \left\{\mid f_{i}(y) \| i \in I\right\} \\
& =p_{I}(x)+p_{I}(y),
\end{aligned}
$$

from the well-known properties of the suprema of subsets of real numbers. Also, about the positive homogeneity of $p_{I}$ we have that

$$
\begin{aligned}
p_{I}(\lambda x) & =\sup \left\{\left|f_{i}(\lambda x)\right| \mid i \in I\right\}=\sup \left\{|\lambda| \mid f_{i}(x) \| i \in I\right\} \\
& =|\lambda| \sup \left\{\left|f_{i}(x)\right| \mid i \in I\right\}=|\lambda| p_{I}(x) .
\end{aligned}
$$

Since $K=\bigcap_{i \in I} k e r f$, then $K \subseteq\left\{x \in E \mid p_{I}(x)=0\right\}_{i}$. For the inverse inclusion, suppose that $p_{I}(y)=0$ for some $y \in E$. Then $\sup \left\{\left|f_{i}(y)\right| \mid i \in I\right\}=0$. The last equality implies

$$
0 \leq\left|f_{i}(y)\right| \leq \sup \left\{\left|f_{i}(y)\right| \mid i \in I\right\}=0,
$$

for each $i \in I$. Then $f_{i}(y)=0$ for each $i \in I$, which implies that $y \in \cap_{i \in I} \operatorname{kerf}_{i}=K$. Then, $p_{I}$ is actually a $K$-deviation measure.

Proposition 2.21. If a $K$-deviation measure is of the form $p_{I}$ indicated in the Example 2.20, it is Lipschitz-continuous.
Proof. According to what is indicated in the Example 2.20

$$
p_{I}(x) \leq\|x\| \text {. }
$$

By subadditivity,

$$
p_{I}(x) \leq p_{I}(x-y)+p_{I}(y),
$$

since $x=(x-y)+y$. By the same way,

$$
p_{I}(y) \leq p_{I}(y-x)+p_{I}(x),
$$

since $y=(y-x)+x$. By the last two inequalities,

$$
p_{I}(x)-p_{I}(y) \leq p_{I}(x-y), p_{I}(y)-p_{I}(x) \leq p_{I}(y-x) .
$$

Since $p_{I}(y-x)=p_{I}(x-y)$ for any $x, y \in E$, this implies

$$
\left|p_{I}(x)-p_{I}(y)\right| \leq p_{I}(x-y) \leq\|x-y\| .
$$

Hence, $p_{I}$ is a Lipschitz-continuous function.

\section{Support Functionals and the Dual Characterization of $K$-Deviation Measures}

In this Section we extend the duality characterization Theorem Theorem 1 of (Rockafellar, Uryasev, \& Zabarankin, 2003) which is proved in the case where the space of financial positions is $L^{2}$ in the case of $K$-deviation measures being defined on Banach spaces.

Theorem 3.1. A functional $D: E \rightarrow[0,+\infty]$ is a lower semicontinuous K-deviation measure if and only if it has a representation of the form

$$
D(x)=\ell(x)-\inf _{f \in F} f(x)=\ell(x)+\sup _{f \in F} f(-x),
$$

where $F \subseteq E^{*}$ is non-empty, weak-star closed and convex, $\ell \in E^{*}$ is a linear functional which corresponds to a "standard premium term" for any $x \in E, K \neq\{0\}$ and $K=\cap_{x^{*} \in F_{D}} k e r x^{*}$, where $F_{D}=\left\{x^{*} \in E^{*} \mid x^{*}=\ell-f, f \in F\right\}$. Also, if we suppose that $E^{*}$ is partially ordered by the cone $P^{0}$, where $P$ is a wedge of $E$, then for any $x \in E \backslash K$ there is some $f_{x} \in F$ such that $\ell(x)>f_{x}(x)$ if $F_{D} \subseteq P_{0}$. Under this dual representation, $F$ is determined by

$$
F=\left\{f \in E^{*} \mid D(x) \geq \ell(x)-f(x), x \in E\right\} .
$$

Also, if $D$ is finite-valued then this is equivalent to the fact that $F$ is bounded.

Proof. Since $D$ is a lower semicontinuous $K$-deviation measure, by Theorem 5.104 of (Aliprantis \& Border, 1999) D is the support functional of the weak-star closed, convex subset of $E^{*}$

$$
F_{D}=\left\{x^{*} \in E^{*} \mid x^{*}(x) \leq D(x), x \in E\right\} .
$$

The last Theorem implies that $D(x)=\sup _{x^{*} \in F_{D}} x^{*}(x)$ for any $x \in E$. Since $D(x)=0$ for any $x \in K$, the last dual representation implies $x^{*}(x)=0$ for any $x^{*} \in F_{D}$. This indicates $x \in$ kerx $^{*}, x^{*} \in F_{D}$. Hence $K \subseteq \cap_{x^{*} \in F_{D}}$ kerx $^{*}$. But also for the inverse inclusion, we get that if $x \in \bigcap_{x^{*} \in F_{D}} \operatorname{ker}^{*}$ then $D(x)=0$, which means that if $x \in K$, then $D(x)=0$. We have that $\cap_{x^{*} \in F_{D}} \operatorname{ker}^{*} \neq\{0\}$ because we suppose that $K \neq\{0\}$. If we suppose that the functional $\ell \in E^{*}$ provides a standard "premium term", we define $F=\left\{f \in E^{*} \mid x^{*}=\ell-f, x^{*} \in F_{D}\right\}$. Then 


$$
F=\left\{f \in E^{*} \mid f=\ell-x^{*}, x^{*} \in F_{D}\right\}
$$

$F$ is also a weak-star closed, convex subset of $E^{*}$. Then in terms of $F$ we also take the following dual representation:

$$
D(x)=\ell(x)+\sup _{f \in F} f(-x)=\ell(x)-\inf _{f \in F} f(x) .
$$

If $F_{D}$ is a bounded set then $F$ is a bounded set and this implies that $D$ is finite-valued, because

$$
D(x)=\sup _{x^{*} \in F_{D}} x^{*}(x) \leq \sup _{x^{*} \in F_{D}}\left\|x^{*}\right\| \cdot\|x\| \leq M \cdot\|x\| \text {, where }
$$

$M>0$ is an upper bound for the norms of the elements of $F_{D}$. Conversely, if $D$ is finite-valued, then since $x^{*}(x) \leq D(x)$ for any $x \in E$, where $x^{*} \in F_{D}$, we get $x^{*}(-x) \leq D(-x)$ and finally $\left|x^{*}(x)\right| \leq \max \{D(x), D(-x)\}$. For any $x^{*} \in F_{D}$ we have

$$
\sup _{x^{*} \in F_{D}}\left|x^{*}(x)\right| \leq \max \{D(x), D(-x)\}<+\infty .
$$

Hence $\sup _{x^{*} \in F_{D}}\left\|x^{*}\right\|<+\infty$ from the Uniform Boundedness Principle and this implies that $F_{D}$ is bounded.

This is actually a characterization of $K$-deviation risk measures defined on a Banach space $E$. For the inverse direction of the proof, suppose that the functional $D: E \rightarrow[0,+\infty]$ with

$$
D(x)=\ell(x)-\inf _{f \in F} f(x)=\ell(x)+\sup _{f \in F} f(-x), x \in E,
$$

where $F \subseteq E^{*}$ is non-empty, weak-star closed. Then $D$ is a lower semicontinuous $K$-deviation measure, where

$K=\bigcap_{x^{*} \in F_{D}} \operatorname{ker}^{*}$ with $K \neq\{0\}$ and

$F_{D}=\left\{x^{*} \in E^{*} \mid x^{*}=\ell-f, f \in F\right\}$. Let us verify the properties of these risk measures:

1) $D(x+k)=\sup _{x^{*} \in F_{D}} x^{*}(x+k)=\sup _{x^{*} \in F_{D}} x^{*}(x)=D(x)$, since $x^{*}(k)=0$ for any $k \in \bigcap_{x^{*} \in F_{D}}$ ker $x^{*}$.

2)

$$
\begin{aligned}
D\left(x+x^{\prime}\right) & =\sup _{x^{*} \in F_{D}} x^{*}\left(x+x^{\prime}\right) \\
& \leq \sup _{x^{*} \in F_{D}} x^{*}(x)+\sup _{x^{*} \in F_{D}} x^{*}\left(x^{\prime}\right)=D(x)+D\left(x^{\prime}\right)
\end{aligned}
$$

from the properties of supremum.

$$
\text { 3) } D(\lambda x)=\sup _{x^{*} \in F_{D}} x^{*}(\lambda x) \leq \sup _{x^{*} \in F_{D}} x^{*}(x)=\lambda D(x) \text { for }
$$

any $\lambda \geq 0$. Also, $D(0)=0$ is obvious.

4) $D(x)=0$ for any $x \in K$, and this holds from the definition of $K$. On the other hand if $x \in E \backslash K$ then there is some $x_{0}^{*} \in F_{D}$ such that $x_{0}^{*}(x) \neq 0$. If $x_{0}^{*}(x)>0$, then we have that $D(x)=\sup _{x^{*} \in F_{D}} x^{*}(x) \geq x_{0}^{*}(x)>0$. If $x \in K$ is such that $x_{0}^{*}(x)<0$, then since also $-x \in E \backslash K$ we have $x_{0}^{*}(-x)>0$ and $D(-x)=\sup _{x^{*} \in F_{D}} x^{*}(-x) \geq x_{0}^{*}(-x)>0$.

Also, $D$ is a lower semicontinuous function defined on $E$ because it is the supremum of a family of lower semicontinuous functions on $E$. The family is the set of linear functionals $x^{*} \in F_{D}$.

\section{The Min-Max Approach on the Risk Minimization for Deviation Risk Measures in $L^{2}$}

In this section we consider the following risk-minimization portfolio-payoff selection problem:

$$
\text { Minimize } \rho(x) \text { subject to } x \in \mathcal{X},
$$

where $\rho$ is a risk measure (not necessarily coherent) and $\mathcal{X}$ is a portfolio-payoff selection set.

The subject of this section is to investigate the saddle-value form of the solution for the problem 1 , if $\rho$ is some deviation measure in the sense defined in (Rockafellar, Uryasev and Zabarankin, 2003).

It is well-known that the portfolio selection problem 1 is a part of the efficient portfolio selection theory and practice, see (Markowiz, 1952), (Kroll, Levy, \& Markowitz, 1984).

We remind that the classic form of a zero-sum game between two players has as payoff function the bilinear form of a dual pair $\left\langle X, X^{*}\right\rangle$ and the strategy set of the one player may be identified by a set $\mathcal{A} \subseteq X$, while the strategy set of the other player may be identified by some $\mathcal{B} \subseteq X^{*}$. The payoff $\left\langle x, x^{*}\right\rangle$ is understood to be a reward paid from the first player to the second. By selecting $x \in \mathcal{A}$, the first players' maximum loss is $\max _{x^{*} \in \mathcal{B}}\left\langle x, x^{*}\right\rangle$. By choosing a proper strategy $x_{0} \in \mathcal{A}$, he may achieve to pay to the second player no more than the minimum of these losses, which is equal to $\mu^{0}=\min _{x \in \mathcal{A}} \max _{x^{*} \in \mathcal{B}}\left\langle x, x^{*}\right\rangle$, if this quantity is well-defined. On the other hand, for any strategy of the second player $x^{*} \in \mathcal{B}$ the minimum payoff he earns is $\min _{x \in \mathcal{A}}\left\langle x, x^{*}\right\rangle$ and by choosing a proper strategy $x^{*} \in \mathcal{B}$, he may achieve to receive from the first player at least the maximum of these earnings, which is equal to

$\mu_{0}=\max _{x^{*} \in \mathcal{B}} \min _{x \in \mathcal{A}}\left\langle x, x^{*}\right\rangle$, if this quantity is well-defined.

$\mu_{0} \leq\left\langle x_{0}, x_{0}^{*}\right\rangle \leq \mu^{0}$ holds and if the equality holds, then the common value is called saddle-value, while the pair

$\left(x_{0}, x_{0}^{*}\right) \in \mathcal{A} \times \mathcal{B}$ which is the solution point of the game, is called saddle-point. We may replace the bilinear form $\langle\cdot, \cdot\rangle$ by another payoff function $F$ defined on $\mathcal{A} \times \mathcal{B}$ and the notions are repeated in the same form. For a brief explanation on zero-sum games which leads to the min-max theorems, see in (Luenberger, 1969). Also, a primal reference for zero-sum games is (von Neumann, 1928). The saddle value

$v=\sup _{x \in \mathcal{A}} \inf _{y \in \mathcal{B}} F(x, y)=\inf _{y \in \mathcal{B}} \sup _{x \in \mathcal{A}} F(x, y)=F(\tilde{x}, \tilde{y})$, can be interpreted as the value of a zero sum game between two players. The one player minimizes $F(x, y)$ over $\mathcal{B}$ supposing that the other player follows the strategy $x$, while the other player maximizes $F(x, y)$ over $\mathcal{A}$ supposing that the other player follows the strategy $y$, see also (Kountzakis, 2011).

We remind the statement of Corollary 3.7 of (Barbu \& Precupanu, 1986) in Paragraph 3.3 of (Barbu \& Precupanu, 1986): If $X, Y$ are reflexive Banach spaces, $\mathcal{A} \subseteq X, \mathcal{B} \subseteq Y$ are bounded, closed and convex sets, $F$ is an upper-lower semicontinuous, concave-convex function on $\mathcal{A} \times \mathcal{B}$, then $F$ has a saddle point on $\mathcal{A} \times \mathcal{B}$, namely a pair $(\tilde{x}, \tilde{y}) \in \mathcal{A} \times \mathcal{B}$ such that

$$
\operatorname{supinf}_{x \in \mathcal{A}} F(x, y)=\inf _{y \in \mathcal{B}} \sup _{x \in \mathcal{A}} F(x, y)=F(\tilde{x}, \tilde{y}) .
$$

Also, we give the following definitions of the payoff functions:

Definition 4.1. A function $F: \mathcal{A} \times \mathcal{B} \rightarrow \overline{\mathbb{R}}$ is concaveconvex like if the following conditions hold:

1) for every $x_{1}, x_{2} \in \mathcal{A}$ and $t \in[0,1]$ there is a $x_{3} \in \mathcal{A}$ such that

$$
t F\left(x_{1}, y\right)+(1-t) F\left(x_{2}, y\right) \leq F\left(x_{3}, y\right)
$$


for all $y \in \mathcal{B}$.

2) for every $y_{1}, y_{2} \in \mathcal{B}$ and $t \in[0,1]$ there is a $y_{3} \in \mathcal{B}$ such that

$$
F\left(x, y_{3}\right) \leq t F\left(x, y_{1}\right)+(1-t) F\left(x, y_{2}\right)
$$

for all $x \in \mathcal{A}$

Definition 4.2. A function $F: \mathcal{A} \times \mathcal{B} \rightarrow \overline{\mathbb{R}}$ is quasi-concaveconvex if the level sets $\left\{x \in \mathcal{A} \mid F\left(x, y_{0}\right) \geq a\right\}$ and $\left\{y \in \mathcal{B} \mid F\left(x_{0}, y\right) \leq a\right\}$ are convex sets for every $x_{0} \in \mathcal{A}, y_{0} \in \mathcal{B}$ and $a \in \mathbb{R}$.

Definition 4.3. A function $F: \mathcal{A} \times \mathcal{B} \rightarrow \overline{\mathbb{R}}$ is called concave-convex if it is concave in the first variable and convex in the second variable.

We remark (see also Remark 3.5 in (Barbu \& Precupanu, 1986)), that a concave-convex function is both concave-convex-like and quasi-concave-convex.

According to Theorem 3 in (Rockafellar, Uryasev, \& Zabarankin, 2003), by considering some set of elements $\mathcal{Q}$ consisted by random variables $Q \in L_{+}^{2}$ such that $\int_{\Omega} Q \mathrm{~d} \mu=1$, or else density functions $\left(Q=\frac{\mathrm{d} f(Q)}{\mathrm{d} \mu}\right.$, where $f(Q)$ denotes the corresponding probability measure), the risk measure $D_{\mathcal{Q}}: L^{2} \rightarrow[0,+\infty]$ with $D_{\mathcal{Q}}(X)=E_{\mu}(X)-\inf \left\{E_{\mu}(X Q) \mid Q \in \mathcal{Q}\right\}$ is a deviation risk measure. $\mathcal{Q}$ is a subset of the base of $L_{+}^{2}$ defined by the constant random variable which is a strictly positive functional of it. The set $\mathcal{Q}$ as it is mentioned in p. 17 of (Rockafellar, Uryasev and Zabarankin, 2003) is considered to be a convex and closed of the base defined by $\mathbf{1}$ on $L_{+}^{2}$. This base is unbounded because $L_{+}^{2}$ induces a lattice ordering on $L^{2}$.

The dual form

$$
D_{\mathcal{Q}}(X)=E_{\mu}(X)-\inf \left\{E_{\mu}(X Q) \mid Q \in \mathcal{Q}\right\}
$$

of a deviation measure $D_{\mathcal{Q}}$ if $\mathcal{Q}$ is convex, closed and bounded and we consider some financial positions' choice set for an investor denoted by $\mathcal{X}$, which has the same properties and it is a subset of $L^{2}$, drives us wonder whether Corollary 3.7 of (Barbu \& Precupanu, 1986) and its game-theoretic implication can be applied in the case of the risk minimization problem. The boundedness of $\mathcal{Q}$ in this case simplifies the saddlevalue solution of the problem.

Actually, we suppose that we have the following version of the risk minimization problem 1:

$$
\text { Minimize } D_{\mathcal{Q}}(X) \text { subject to } X \in \mathcal{X}
$$

Apart from the Proposition 2 in (Rockafellar, Uryasev, \& Zabarankin, 2003) which indicates that finite-valued deviation measures on $L^{2}$ being lower semicontinuous are norm-continuous, we prove a stronger result than Proposition 2 in (Rockafellar, Uryasev, \& Zabarankin, 2003), since it indicates that they are Lipschitz continuous in the case we consider.

Proposition 4.4. Any deviation measure $D_{\mathcal{Q}}: L^{2} \rightarrow[0,+\infty)$, where $\mathcal{Q}$ is a convex and bounded subset of $L_{+}^{2}$ such that $E_{\mu}(Q)=1$ is a Lipschitz function.

Proof. We have to prove that $D_{\mathcal{Q}}$ is a norm-continuous function on $L^{2}$. But if $\mathcal{Q}$ is a norm-bounded set, this implies that $D_{\mathcal{Q}}$ is a Lipschitz function. This is true because for two families of functions $f_{i}, g_{i}: L^{2} \rightarrow \mathbb{R}$ such that

$$
\begin{aligned}
\sup \left\{f_{i}(X)+g_{i}(Y) \mid i \in I\right\} & <+\infty, \sup \left\{f_{i}(X) \mid i \in I\right\} \\
& <+\infty, \sup \left\{g_{i}(Y) \mid i \in I\right\}<+\infty
\end{aligned}
$$

and for any $X, Y \in L^{2}$ that satisfy the above finite suprema conditions,

$$
\sup \left\{f_{i}(X)+g_{i}(Y) \mid i \in I\right\} \leq \sup \left\{f_{i}(X) \mid i \in I\right\}+\sup \left\{g_{i}(Y) \mid i \in I\right\}
$$

is true. Hence if $I=\mathcal{Q}, \quad f_{Q}(X)=g_{Q}(X)=E_{\mu}(X(1-Q))$ and since $X=(X-Y)+Y$ for any $X, Y \in L^{2}$, this implies

$$
\begin{aligned}
D_{\mathcal{Q}}(X) & =\sup \left\{E_{\mu}(X(1-Q)) \mid Q \in \mathcal{Q}\right\} \\
& \leq \sup \left\{E_{\mu}((X-Y)(1-Q)) \mid Q \in \mathcal{Q}\right\} \\
& +\sup \left\{E_{\mu}(Y(1-Q)) \mid Q \in \mathcal{Q}\right\} \\
& =D_{\mathcal{Q}}(X-Y)+D_{\mathcal{Q}}(Y) .
\end{aligned}
$$

By the same way we have that

$$
D_{\mathcal{Q}}(Y) \leq D_{\mathcal{Q}}(Y-X)+D_{\mathcal{Q}}(X),
$$

since $Y=(Y-X)+X$. Finally,

$D_{\mathcal{Q}}(X)-D_{\mathcal{Q}}(Y) \leq E_{\mu}\left((X-Y)\left(1-Q_{1}\right)\right)$, where $Q_{1} \in \mathcal{Q}$ because since $\mathcal{Q}$ is convex, closed and bounded subset of a reflexive space, it is a weakly compact subset of it and the supremum in $D_{\mathcal{Q}}(X-Y)$ is actually a maximum. Hence we consider $Q_{1}$ to be a maximizer of $(X-Y)$ over $\mathcal{Q}$. In the same way, $D_{\mathcal{Q}}(Y)-D_{\mathcal{Q}}(X) \leq E_{\mu}\left((Y-X)\left(1-Q_{2}\right)\right)$, for some $Q_{2} \in \mathcal{Q}$. Hence,

$$
\begin{aligned}
\left|E_{\mu}((X-Y)(1-Q))\right| & \leq\|X-Y\|_{2}\|1-Q\|_{2} \leq\|X-Y\|_{2}\left(1+\|Q\|_{2}\right) \\
& \leq\|X-Y\|_{2}(1+m),
\end{aligned}
$$

for some upper bound of the norms of the elements of $\mathcal{Q}$.

Proposition 4.5. If we suppose that $\mathcal{Q}$ and $\mathcal{X}$ are convex, closed and bounded, the problem 2 has a solution.

Proof. Since $D_{\mathcal{Q}}$ is a norm-continuous function, then the problem 2 has a solution, since $D_{\mathcal{Q}}$ is also weakly lower semicontinuous and $\mathcal{Q}$ is a weakly compact set.

Since the problem 2 has a solution, it has an optimal value. We will investigate whether this optimal value is a saddle value, according to Corollary 3.7 of (Barbu \& Precupanu, 1986).

The duality form of $D_{\mathcal{Q}}$, implies that the candidate twovariable function for the application of Corollary 3.7 of (Barbu \& Precupanu, 1986) is $F: \mathcal{Q} \times \mathcal{X} \rightarrow \mathbb{R}$, where

$$
F(Q, X)=E_{\mu}(X(1-Q)), Q \in \mathcal{Q}, X \in \mathcal{X} .
$$

For this function we have the following.

Proposition 4.6. The function $F: \mathcal{Q} \times \mathcal{X} \rightarrow \mathbb{R}$ satisfies the properties of Corollary 3.7 of (Barbu \& Precupanu, 1986), hence the optimal value of the risk minimization problem 2 is a value of the function $F$, namely

$$
\inf \left\{D_{\mathcal{Q}}(X) \mid X \in \mathcal{X}\right\}=F\left(Q_{0}, X_{0}\right)
$$

for some $X_{0} \in \mathcal{X}, Q_{0} \in \mathcal{Q}$.

Proof. $F$ is upper-lower semicontinuous, because it is norm-continuous in both of its variables. Moreover, it is linear in both of its variables, which implies that it is concave-convex. Hence the conclusion is true from Corollary 3.7 of (Barbu \& Precupanu, 1986).

The economic interpretation of the fact that the risk minimization problem is solved through determining a saddle-point of the function $F$ is the following: The minimization of risk corresponds to a zero-sum game between the investor and the market. The payoff function of the game - the one which is 
minimized by the investor as a cost function for a given "valuation" density $Q$ over the set of financial positions $\mathcal{X}$ is the partial function $F(Q, \cdot)$. The function being maximized as a "value" function for a specific financial position $X \in \mathcal{X}$ by the market over the set of valuation densities $\mathcal{Q}$ is the partial function $F(\cdot, X)$. The value of the game, which is also the optimal value of the risk minimization problem 2 is achieved at a saddle point $\left(Q_{0}, X_{0}\right)$. This meets the notion of a "two-person zero-sum game" for one more reason, because the market can be viewed as a whole to which the monetary cost of the risk minimization is paid (the one player) and the investor can be viewed as the other player who earns the monetary payoff concerning a certain financial position $X$, which is formulated by the market as the value of it. To be more accurate, suppose that the set of strategies of the market is the set of the valuation measures $\mathcal{Q}$, while the set of strategies of the investor is the set of the financial positions $\mathcal{X}$. If we select some $X \in \mathcal{X}$, the investor's maximum loss is $\max _{Q \in \mathcal{Q}} F(Q, X)$. By choosing a proper strategy $X_{1} \in \mathcal{X}$, she may achieve to pay to the second player (to the market) no more than the minimum of the above costs, being $\mu^{1}=\min _{X \in \mathcal{X}} \max _{Q \in \mathcal{Q}} F(Q, X)$, if this quantity is well-defined. On the other hand, the market for any strategy $Q \in \mathcal{Q}$ of it, the minimum payoff that it earns from the investor is $\min _{X \in \mathcal{X}} F(Q, X)$ and by choosing a proper strategy $Q_{1} \in \mathcal{Q}$, it may achieve to receive from the investor at least the maximum of these earnings which is

$\mu_{1}=\max _{Q \in \mathcal{Q}} \min _{X \in \mathcal{X}} F(Q, X)$, if this quantity is well-defined. If there is a $\left(Q_{0}, X_{0}\right) \in \mathcal{Q} \times \mathcal{X}$ such that $\mu_{1}=\mathbb{E}_{Q_{0}}\left(X_{0}\right)=\mu^{1}$, then $X_{0}$ is a solution to the deviation minimization problem 2 . For a similar explanation on saddle-value form that minimization of convex risk measures may take, see also in (Kountzakis, 2011).

\section{The Risk Minimization for Deviation Measures on Reflexive Spaces: Bounded Sets}

In this section we prove the existence of solution to the problem of minimization of deviation if the deviation measure comes from a certain class of coherent risk measures.

Specifically, if we transfer the above results to the frame of the commodity-price duality $\left\langle E, E^{*}\right\rangle$, where the space $E$ denotes a reflexive space in which the financial positions lie in, then we get a saddle-point solution result for the following minimization problem

$$
\text { Minimize } D_{\rho_{(p, e)}}(x) \text { subject to } x \in \mathcal{X},
$$

where $\mathcal{X}$ is a convex, closed, bounded subset of $E, P$ is closed and int $P \neq \varnothing$ and $e \in \operatorname{int} P, \ell \in E^{*}$ and $\rho_{(P, e)}: E \rightarrow(-\infty,+\infty)$ denotes a $(P, e)$-coherent risk measure on $E$. The closed subspace $K$ is such that for any $x \in K$, $\pi(-x)=\ell(-x), x \in K$ holds for any $\pi \in B$, while for any $x \in E \backslash K$, there is a $\pi_{x} \in B$ such that $\pi_{x}(-x)>\ell(-x)$. Also $B=B_{e} \cap \mathcal{A}_{\rho_{(P, e)}}^{0}$. The functional $D_{\rho_{(P, e)}}: E \rightarrow[0,+\infty)$ is defined as follows:

$$
D_{\rho_{(p, e)}}(x)=\sup \{\pi(-x) \mid \pi \in B\}+\ell(x),
$$

where $B=\mathcal{A}_{\rho}^{0} \cap B_{e}$ and $B_{e}$ is the base defined by $e$ on $P^{0} . \mathcal{A}_{\rho_{(P, e)}}^{0}$ is the dual wedge of $\mathcal{A}_{\rho_{(P, e)}}$ in $E^{*}$.

Theorem 4.7. The problem 3 has a solution via saddlepoints.
Proof. According to the above dual representation for $D_{\rho_{(P, e)}}$, we get that

$$
D_{\rho_{(P, e)}}(x)=\sup \{-\pi(x)+\ell(x) \mid \pi \in B\}, x \in \mathcal{X} .
$$

In order to apply Corollary 3.7 of (Barbu \& Precupanu, 1986) in this case, we have to determine the payoff function $F: \mathcal{A} \times \mathcal{B} \rightarrow \mathbb{R}$, where $\mathcal{A} \subseteq X, \mathcal{B} \subseteq Y$ are convex, closed and bounded subsets of the reflexive spaces $X, Y$ and $F$ has to be a concave-convex and upper-lower semicontinuous function. We notice that $Y=E, X=E^{*}, \mathcal{B}=\mathcal{X}, \mathcal{A}=B$ and

$F: B \times \mathcal{X} \rightarrow \mathbb{R}$ with $F(\pi, x)=-\pi(x)+\ell(x) . F$ is concaveconvex and upper-lower semicontinuous. Then a saddle-point $\left(\pi_{0}, x_{0}\right) \in B \times \mathcal{X}$ exists, or else

$$
F\left(\pi_{0}, x_{0}\right)=\operatorname{supinf}_{\pi \in B} F(\pi, x)=\inf _{x \in \mathcal{X}} \sup _{\pi \in B} F(\pi, x)=\inf _{x \in \mathcal{X}} D_{\rho_{(P, e)}}(x) .
$$

According to the saddle-point conditions for $\left(\pi_{0}, x_{0}\right)$, $F\left(\pi_{0}, x_{0}\right)=D_{\rho_{(P, e)}}\left(x_{0}\right)$.

\section{The Minimization of Deviation Measures in Banach Spaces: Unbounded Sets}

The question which arises is whether the above min-max approach for the minimization of deviation measures can be generalized in the case of an unbounded choice set of financial (risk) positions. The answer is affirmative due to an alternative min-max theorem reminded in p. 10 of (Delbaen, 2002). We also focus on the classes of deviation measures related to the coherent measures arising from ordering cones with non-empty interior.

Specifically, the statement of the previously mentioned min-max theorem is the following: Let $K$ be a compact, convex subset of a locally convex space $Y$. Let $L$ be a convex subset of an arbitrary vector space $X$. Suppose that $u$ is a bilinear function $u: X \times Y \rightarrow \mathbb{R}$. For each $l \in L$, we suppose that the partial (linear) function $u(l, \cdot)$ is continuous on $Y$. Then we have that

$$
\inf _{l \in L} \sup _{k \in K} u(l, k)=\operatorname{supinf}_{k \in K} u(l, k) .
$$

Then we have the following

Theorem 5.1. Suppose that $E$ is a reflexive space. Consider the problem

$$
\text { Minimize } D_{\rho_{(p, e)}}(x) \text { subject to } x \in \mathcal{X},
$$

where $\mathcal{X}$ is a convex, unbounded subset of $E, P$ is closed and $\operatorname{int} P \neq \varnothing$, and $e \in \operatorname{int} P, \ell \in E^{*}$. The closed subspace $K$ is such that for any $x \in K, \pi(-x)=\ell(-x), x \in K$ holds for any $\pi \in B$, while for any $x \in E \backslash K$, there is a $\pi_{x} \in B_{e}$ such that $\pi_{x}(-x)>\ell(-x)$. The functional $D_{\rho_{(P, e)}}: E \rightarrow[0,+\infty)$ is defined as follows:

$$
D_{\rho_{(P, e)}}(x)=\sup \{\pi(-x) \mid \pi \in B\}+\ell(x),
$$

where $B=\mathcal{A}_{\rho_{(P, e)}}^{0} \cap B_{e}$ and $B_{e}$ is the base defined by $e$ on $P^{0}$. Then the problem 4 has a solution.

Proof. If we apply the previous min-max theorem, we have that $Y=E^{*}$ endowed with the weak topology, $X=E$, $K=B, L=\mathcal{X} . Y$ is a locally convex space, $E$ is a linear space, $u: E \times E^{*} \rightarrow \mathbb{R}, \quad u(x, \pi)=\ell(x)-\pi(x), x \in E, \pi \in E^{*}$. The functional $\ell \in E^{*}$ is the one specified by assumptions. Also, for any $x \in \mathcal{X}$, the partial function 


\section{E. KOUNTZAKIS}

$u(x, \cdot)=u_{x}: E^{*} \rightarrow \mathbb{R}$ where $u_{x}(\pi)=\ell(x)-\pi(x)$ for any $x \in E$ and hence for any $x \in \mathcal{X}$. The partial function $u_{x}$ is weakly continuous, since if we consider a net $\left(\pi_{a}\right)_{a \in A} \subseteq E^{*}$

such that $\pi_{a} \stackrel{\sigma\left(E^{*}, E^{* *}\right)}{\longrightarrow} \pi$, for the specific $x \in E$,

$\pi_{a}(x) \rightarrow \pi(x)$, then we get

$u\left(x, \pi_{a}\right)=\ell(x)+\pi_{a}(-x) \rightarrow u(x, \pi)=\ell(x)+\pi(-x)$. Finally, $u_{x}\left(\pi_{a}\right) \rightarrow u_{x}(\pi)$ for each $x \in E$ and for each specific $x \in \mathcal{X}$. This implies that any partial function $u_{x}, x \in E$ is weakly continuous, which is also valid for any $x \in \mathcal{X}$. Also, $u$ is a bilinear function as it arises from its definition. Since the base $B_{e}$ of the cone $P$ is convex and weakly compact, the set $B$ is weakly compact and convex, too. Also, the set $\mathcal{X}$ is convex and the conditions for the validity of the conclusion of the previous min-max theorem hold. Hence the minmax equation holds for $u$, which implies the existence of a saddle-point $\left(x_{1}, \pi_{1}\right) \in \mathcal{X} \times B$ such that

$$
\begin{aligned}
\inf _{x \in \mathcal{X}} D_{\rho_{(P, e)}}(x) & =\inf _{x \in \mathcal{X}} \sup _{\pi \in B} u(x, \pi)=\operatorname{supinf}_{\pi \in B} u(x, \pi) \\
& =u\left(x_{1}, \pi_{1}\right)=D_{\rho_{(P, e)}}\left(x_{1}\right) .
\end{aligned}
$$

The existence of a saddle-point is implied by Proposition 3.1 of (Barbu \& Precupanu, 1986) which says that a function satisfies the min-max equality if and only if it has a saddlepoint.

We prove the corresponding Theorem for non-reflexive Banach spaces.

Theorem 5.2. Suppose that $E$ is a non-reflexive Banach space. Consider the problem

$$
\text { Minimize } D_{\rho_{\left(\eta_{0}, e\right)}}(x) \text { subject to } x \in \mathcal{X},
$$

where $\mathcal{X}$ is a convex, unbounded subset of $E$. If $P$ is a closed cone of $E^{*}$ and $\operatorname{int} P_{0} \neq \varnothing$ and $e \in \operatorname{int} P_{0}, \quad \ell \in E^{*}$ and $\rho_{\left(P_{0}, e\right)}: E \rightarrow(-\infty,+\infty)$ denotes a $\left(P_{0}, e\right)$-coherent risk measure on $E$. The closed subspace $K$ is such that for any $x \in K, \pi(-x)=\ell(-x), x \in K$ holds for any $\pi \in B_{e}^{*}$, while for any $x \in E \backslash K$, there is a $\pi_{x} \in B_{e}^{*}$ such that

$\pi_{x}(-x)>\ell(-x)$. Then the functional $D_{\rho_{\left(\mathrm{f}_{0}, e\right)}}: E \rightarrow[0,+\infty)$, where

$$
D_{\rho_{(\mathrm{P}, e)}}(x)=\sup \left\{\pi(-x) \mid \pi \in B_{1}\right\}+\ell(x),
$$

is a $K$-deviation risk measure, where $B_{1}=\mathcal{A}_{\rho}^{0} \cap B_{e}^{*}$ and $B_{e}^{*}$ is the base defined by $e$ on $P$. Then the problem 5 has a solution.

Proof. If we apply the previous min-max theorem, we have that $Y=E^{*}$ endowed with the weak-star topology, $X=E$, $K=B_{1}, L=\mathcal{X} . Y$ is a locally convex space, $E$ is a linear space, $u: E \times E^{*} \rightarrow \mathbb{R}, \quad u(x, \pi)=\ell(x)-\pi(x), x \in E, \pi \in E^{*}$. The functional $\ell \in E^{*}$ is the one specified by assumptions. $E$ is a linear space, $u: E \times E^{*} \rightarrow \mathbb{R}$,

$u(x, \pi)=\ell(x)-\pi(x), x \in E, \pi \in E^{*}$. Also, for any $x \in \mathcal{X}$, the partial function $u(x, \cdot)=u_{x}: E^{*} \rightarrow \mathbb{R}$ where

$u_{x}(\pi)=\ell(x)-\pi(x)$ for any $x \in E$ and hence for any

$x \in \mathcal{X}$. The partial function $u_{x}$ is weak-star continuous, since

if we consider a net $\left(\pi_{a}\right)_{a \in A} \subseteq E^{*}$ such that $\pi_{a} \stackrel{\sigma\left(E^{*}, E\right)}{\longrightarrow} \pi$, for the specific $x \in E, \pi_{a}(x) \rightarrow \pi(x)$, then we get $u\left(x, \pi_{a}\right)=\ell(x)+\pi_{a}(-x) \rightarrow u(x, \pi)=\ell(x)+\pi(-x)$. Finally, $u_{x}\left(\pi_{a}\right) \rightarrow u_{x}(\pi)$ for each $x \in E$ and for each specific

$x \in \mathcal{X}$. This implies that any partial function $u_{x}, x \in E$ is weak-star continuous, which is also valid for any $x \in \mathcal{X}$. Also, $u$ is a bilinear function as it arises from its definition. Also, since the base $B_{e}^{*}$ is weak-star compact and convex base of the cone $P$, then the set $B_{1}$ is a weak-star compact and convex subset of $E^{*}$ and the set $\mathcal{X}$ is a convex subset of $E$, then the conditions for the validity of the conclusion of the previous min-max theorem hold. Hence the min-max equation holds for $u$, which implies the existence of a saddle-point $\left(x_{2}, \pi_{2}\right) \in \mathcal{X} \times B_{1}$ such that

$$
\begin{aligned}
\inf _{x \in \mathcal{X}} D_{\rho_{\left(P_{0}, e\right)}}(x) & =\inf _{x \in \mathcal{X}} \sup _{\pi \in B_{1}} u(x, \pi)=\operatorname{supinf}_{\pi \in B_{1}} u(x \in \mathcal{X} \\
& =u\left(x_{2}, \pi_{2}\right)=D_{\rho_{\left(\Gamma_{0}, e\right)}}\left(x_{2}\right) .
\end{aligned}
$$

The existence of a saddle-point is implied by Proposition 3.1 of (Barbu \& Precupanu, 1986) which says that a function satisfies the min-max equality if and only if it has a saddle-point.

Remark 5.3. We remind for the sake of completeness of what we proved in the last two Theorems that the fact that if $e \in$ intP then $P^{0}$ has a $\sigma\left(E^{*}, E\right)$-compact base is mentioned in Proposition 13.8.12 in (Jameson, 1970). The weakstar compactness of bases defined by elements of $E$ on cones of $E^{*}$ is implied in non-reflexive spaces from the proposition Proposition 2.4 of (Kountzakis, 2011), which is actually a reference to Theorem 39 of (Xanthos, 2009).

\section{Minimization of the Usual Deviation Measure Arising from $E S_{a}$}

Expected shortfall $E S_{a}$ is identical to $C V a R_{a}$ as Corollary 4.3 in (Acerbi \& Tasche, 2002) indicates. $C V a R_{a}$ is initially defined in Definition 2.5 of (Acerbi \& Tasche, 2002), while $E S_{a}$ is a coherent risk measure on $L^{1}(\Omega, \mathcal{F}, \mu)$ (see Proposition 3.1 in (Acerbi \& Tasche, 2002)). As it is quoted in (Acerbi $\&$ Tasche, 2002), the expression $E S_{a}(x)=-\frac{1}{a} \int_{0}^{a} q_{u}(x) \mathrm{d} u$ indicates that $E S_{a}$ is the building block for law invariant, coherent risk measures, according to the results containing in (Kusuoka, 2001). These properties of $\mathrm{CVaR}_{a}$ may make it very attractive in applications, since it could replace $V_{a} R_{a}$. Also, as it is mentioned in (Rockafellar, Uryasev, \& Zabarankin, 2003), a shortfall relative to expectation is more adequate in practice. A very interesting application of the saddle-point method in order to verify the existence of solution to the minimization of deviation risk is also by the use of min-max Theorem mentioned in p. 10 of (Delbaen, 2002) in the case of the "deviation which arises from expeced shortfall", which is defined as the functional $D: L^{1}(\Omega, \mathcal{F}, \mu) \rightarrow[0,+\infty)$ with $D_{a}(x)=E S_{a}(x)+\mathbb{E}_{\mu}(x), x \in L^{1}(\Omega, \mathcal{F}, \mu)$ for a level of significance $a \in(0,1)$. As it is well-known from Acerbi and Tasche, 2002) and (Tasche, 2002) the expected shortfall $E S_{a}(x)$ for a financial position and a level of significance $a \in(0,1)$ is defined in Definition 2.6 of (Acerbi \& Tasche, 2002) as the negative of tail-mean of $x$ at the level $a$, being equal to

$$
E S_{a}(x)=-\frac{1}{a}\left(E\left(x \mathbf{1}_{\left\{x \leq q_{a}(x)\right\}}\right)-q_{a}(x)\left(a-\mu\left(x \leq q_{a}(x)\right)\right)\right),
$$

where $q_{a}(x)$ denotes the $a$-lower quantile of $x$. The deviation measure $D$ is introduced in Example 4 of (Rockafellar, Uryasev, \& Zabarankin, 2003). Also, expected shor- 


\section{E. KOUNTZAKIS}

tfall according to Theorem 4.1 of (Kaina \& Rüschendorf, 2009) admits the dual representation

$$
E S_{a}(x)=\max _{Q \in Z_{a}} \mathbb{E}_{Q}(-x),
$$

where $\mathcal{Z}_{a}=\left\{Q \in M_{1} \mid \frac{\mathrm{d} Q}{\mathrm{~d} \mu} \leq \frac{1}{a}, \mu\right.$-a.e. $\} . M_{1}$ denotes the set of $\mu$-continuous probability measures on the measurable space $(\Omega, \mathcal{F}) . \frac{\mathrm{d} Q}{\mathrm{~d} \mu} \in L^{1}(\Omega, \mathcal{F}, \mu)$. But for the probability measures of the representation set $\mathcal{Z}_{a}, \frac{\mathrm{d} Q}{\mathrm{~d} \mu} \in\left[0, \frac{1}{a} \mathbf{1}\right]$ holds, with respect to the usual (pointwise) partial ordering on $L^{\infty}(\Omega, \mathcal{F}, \mu)$. This implies $\frac{\mathrm{d} Q}{\mathrm{~d} \mu} \in L^{\infty}(\Omega, \mathcal{F}, \mu)$ for any $Q \in \mathcal{Z}_{a}$.

Lemma 5.4. $\mathcal{Z}_{a}=\left\{\frac{\mathrm{d} Q}{\mathrm{~d} \mu} \mid Q \in \mathcal{Z}_{a}\right\}$ is a weak-star compact set of $L^{\infty}(\Omega, \mathcal{F}, \mu)$.

Proof. We consider the set

$$
\mathcal{Z}_{a}=\left\{Q \in \operatorname{ca}(\Omega) \mid Q(\Omega)=1, Q \ll \mu, \frac{\mathrm{d} Q}{\mathrm{~d} \mu} \in \mathcal{D}_{a}\right\},
$$

and $\mathcal{D}_{a}$ is the order-interval $\mathcal{D}_{a}=\left[0, \frac{1}{a} \mathbf{1}\right]$ of $L^{\infty}$ which is $\sigma\left(L^{\infty}, L^{1}\right)$-compact due to Lemma 7.54 of (Aliprantis \& Border, 1999). We also have to prove that $Z_{a}$ is weak-star closed in $L^{\infty}$. Let us consider a net $\left(Q_{\lambda}\right)_{\lambda \in \Lambda} \subseteq Z_{a}$ such that

$$
\frac{\mathrm{d} Q_{\lambda}}{\mathrm{d} \mu} \stackrel{\sigma\left(L^{\infty}, L^{1}\right)}{\longrightarrow} f .
$$

From the fact that $\frac{\mathrm{d} Q_{\lambda}}{\mathrm{d} \mu}, \lambda \in \Lambda$, we obtain that $\frac{\mathrm{d} Q_{\lambda}}{\mathrm{d} \mu} \in L^{1}(\Omega, \mathcal{F}, \mu)$. We have to prove that $f$ is a RadonNikodym derivative of some measure $Q_{1} \in \mathcal{Z}_{a}$ with respect to $\mu$. Let us consider the map $Q_{1}: \mathcal{F} \rightarrow[0,1]$ where

$$
Q_{1}(A)=\int_{\Omega} f \cdot I_{A} \mathrm{~d} \mu
$$

and $I_{A}$ is the characteristic random variable of $A$. In order to show that $Q_{1}$ is a probability measure,

$$
Q_{1}(\Omega)=\int_{\Omega} f \mathrm{~d} \mu
$$

which is the limit $\lim _{\lambda \in \Lambda} \int_{\Omega} \mathrm{d} Q_{\lambda}$ and every of the terms of the net of real numbers

$$
\left(\int_{\Omega} \mathrm{d} Q_{\lambda}\right)_{\lambda \in \Lambda}
$$

is equal to 1 . By the same argument, we may deduce that $Q_{1}(\varnothing)=0$. If $\left(A_{n}\right)_{n \in \mathbb{N}}$ is a sequence of sets in $\mathcal{F}$ which are disjoint, then

$$
Q_{\lambda}\left(\cup_{k=1}^{n} A_{k}\right)=\sum_{k=1}^{n} Q_{\lambda}\left(A_{k}\right), \lambda \in \Lambda .
$$

Hence,

$$
Q_{1}\left(\cup_{k=1}^{n} A_{k}\right)=\sum_{k=1}^{n} Q_{1}\left(A_{k}\right), n \in \mathbb{N},
$$

where $\mathbb{N}$ denotes the set of natural numbers. For $n \rightarrow \infty$

$$
Q_{1}\left(\cup_{n=1}^{\infty} A_{n}\right)=\sum_{n=1}^{\infty} Q_{1}\left(A_{n}\right),
$$

from the $\sigma\left(L^{\infty}, L^{1}\right)$-convergence

$$
\frac{\mathrm{d} Q_{\lambda}}{\mathrm{d} \mu} \stackrel{\sigma\left(L^{\infty}, L^{1}\right)}{\longrightarrow} f,
$$

and the definition of $Q_{1}$, the fact that any characteristic function $I_{A}, A \in \mathcal{F}$ belongs to $L^{1}(\Omega, \mathcal{F}, \mu)$. We may also refer to the Monotone Convergence Theorem (11.17 in (Aliprantis $\&$ Border, 1999), where the restriction of the $f$ on the set $\cup_{n=1}^{\infty} A_{n}$ is the integrable function which is mentioned in the Theorem, while $f_{n}$ is the restriction of $f$ on a set of the form $\cup_{k=1}^{n} A_{k}$. For the $\mu$-continuity of $Q_{1}$, we have that if for a set $A \in \mathcal{F} \quad \mu(A)=0$ holds, then since $Q_{\lambda}, \lambda \in \Lambda$ is $\mu$-continuous,

$$
Q_{\lambda}(A)=0=\int_{A} \frac{\mathrm{d} Q_{\lambda}}{\mathrm{d} \mu} \mathrm{d} \mu,
$$

for any $\lambda \in \Lambda$. But since

$$
\frac{\mathrm{d} Q_{\lambda}}{\mathrm{d} \mu} \stackrel{\sigma\left(L^{\infty}, L^{1}\right)}{\longrightarrow} f,
$$

then

$$
Q_{1}(A)=\int_{A} f \mathrm{~d} \mu=\lim _{\lambda \in \Lambda} \int_{A} \frac{\mathrm{d} Q_{\lambda}}{\mathrm{d} \mu} \mathrm{d} \mu=0 .
$$

Hence $Q_{1}$ is $\mu$-continuous. Since $Q_{\lambda}, \lambda \in \Lambda$ are probability measures,

$$
\frac{\mathrm{d} Q_{\lambda}}{\mathrm{d} \mu}(\omega) \geq 0
$$

$\mu$-a.e. Also, since $Q_{1}$ is a $\mu$-continuous probability measure, by Radon-Nikodym Theorem we have

$$
\frac{\mathrm{d} Q_{1}}{\mathrm{~d} \mu}=f,
$$

$\mu$-a.e. and $f(\omega) \geq 0, \quad \mu$-a.e. In order to show that

$$
0 \leq f \leq \frac{1}{a} \mathbf{1}
$$

with respect to the usual (point-wise) partial ordering on $L^{\infty}(\Omega, \mathcal{F}, \mu)$, we use the convergence argument

$$
\int_{A} \frac{\mathrm{d} Q_{\lambda}}{\mathrm{d} \mu} \mathrm{d} \mu \rightarrow \int_{A} f \mathrm{~d} \mu
$$

for any $A \in \mathcal{F}$. This implies that $\int_{A} f \mathrm{~d} \mu \in\left[0, \frac{1}{a}\right]$ for any $A \in \mathcal{F}$. This implies $0 \leq f \leq \frac{1}{a} \mathbf{1} \quad \mu$-a.e., since if we suppose that this does not hold, then there exists some $B \in \mathcal{F}$ with $\mu(B)>0$ such that either $f(\omega)>\frac{1}{a}$, or $f(\omega)<0$ for any $\omega \in B$. Then, we would have either $\int_{B} f \mathrm{~d} \mu>\frac{1}{a}$, or $\int_{B} f \mathrm{~d} \mu<0$, a contradiction. Finally, the set $\mathcal{Z}_{a}$ is a weak-star closed subset of a weak-star compact set which is the set $\mathcal{D}_{a}$.

The above deviation measure $D_{a}$ is denoted by $C V a R_{a}^{\Delta}$ in 
(Rockafellar, Uryasev, \& Zabarankin, 2003), see p. 7 in (Rockafellar, Uryasev, \& Zabarankin, 2003).

Hence, we have the following risk minimization problem

$$
\text { Minimize } C \operatorname{VaR}_{a}^{\Delta}(x) \text { subject to } x \in \mathcal{X}
$$

The existence of solution to the risk minimization problem 6 does not depend on the fact whether the set of financial positions $\mathcal{X}$ which is the selection set of the investor is bounded or not.

Theorem 5.5. If $\mathcal{X}$ is a convex set of $L^{1}(\Omega, \mathcal{F}, \mu)$, then the deviation risk minimization problem 6 has a solution.

Proof. We will apply the min-max theorem reminded in p. 10 of (Delbaen, 2002). We have that $Y=L^{\infty}$ endowed with the weak-star topology, $X=L^{1}, K=\mathcal{D}_{a}, L=\mathcal{X} . Y$ is a locally convex space, $X=L^{1}$ is a linear space, $u: L^{1} \times L^{\infty} \rightarrow \mathbb{R}$ $u(x, \pi)=\mathbb{E}_{\mu}(x)+\pi(-x), x \in L^{1}, \pi \in L^{\infty}$. We notice that $u$ is a bilinear function over the product of the spaces defined and this arises by its definition. The partial function $u(x, \cdot)$ is actually the function $u_{x}: L^{\infty} \rightarrow \mathbb{R}$, where

$u_{x}(\pi)=\mathbb{E}_{\mu}(x)+\pi(-x)=\mathbf{1}(x)-\pi(x)$. This function is weakstar continuous for any $x \in L^{1}$ and consequently for any $x \in \mathcal{X}$. By 1 we denote the random variable for which $\mathbf{1}(\omega)=1, \omega \in \Omega$. Suppose that $\left(\pi_{\lambda}\right)_{\lambda \in \Lambda}$ is a net in $L^{\infty}$, which is $\sigma\left(L^{\infty}, L^{1}\right)$-convergent to some $\pi \in L^{\infty}$. Hence for any $x \in L^{1}$ and of course for any specific $x \in \mathcal{X}, \pi_{\lambda}(x) \rightarrow \pi(x)$. Also, $\pi_{\lambda}(-x) \rightarrow \pi(-x)$ and finally

$u\left(x, \pi_{\lambda}\right)=\mathbb{E}_{\mu}(x)+\pi_{\lambda}(-x) \rightarrow \mathbb{E}_{\mu}(x)+\pi(-x)=u(x, \pi)$. But $x$ is specified, hence $u_{x}\left(\pi_{\lambda}\right) \rightarrow u_{x}(\pi)$ which implies the weak-star continuity of the partial function $u_{x}: L^{\infty} \rightarrow \mathbb{R}$. Hence since $\mathcal{X}$ is a convex subset of $L^{1}$ and $\mathcal{D}_{a}$ is a weak-star compact, convex subset of $L^{\infty}$, all the conditions of the min-max Theorem reminded in p.10 of (Delbaen, 2002) are valid. Hence the min-max equation holds for $u$, which implies the existence of a saddle-point $\left(x_{*}, \pi_{Q^{*}}\right) \in \mathcal{X} \times \mathcal{D}_{a}$ such that

$$
\begin{aligned}
\inf _{x \in \mathcal{X}} \operatorname{CVaR}_{a}^{\Delta}(x) & =\inf _{x \in \mathcal{X}} \sup _{\pi_{Q} \in \mathcal{D}_{a}} u\left(x, \pi_{Q}\right)=\sup _{\pi_{Q} \in \mathcal{D}_{a}} \inf _{x \in \mathcal{X}} u\left(x, \pi_{Q}\right) \\
& =u\left(x_{*}, \pi_{Q^{*}}\right)=\operatorname{CVaR}_{a}^{\Delta}\left(x_{*}\right) .
\end{aligned}
$$

The existence of a saddle-point is implied Proposition 3.1 of (Barbu \& Precupanu, 1986) which says that a function satisfies the min-max equality if and only if it has a saddle-point.

\section{REFERENCES}

Acerbi, C., \& Tasche, D. (2002). On the coherence of the expected shortfall. Journal of Banking and Finance, 26, 1487-1503. doi:10.1016/S0378-4266(02)00283-2

Aliprantis, C. D., \& Border, K. C. (1999). Infinite dimensional analysis, A hitchhiker's guide (2nd ed.). New York: Springer. doi:10.1007/978-3-662-03961-8

Artzner, P., Delbaen, F., Eber, J. M., \& Heath, D. (1999). Coherent measures of risk. Mathematical Finance, 9, 203-228. doi:10.1111/1467-9965.00068

Barbu, V., \& Precupanu, T. (1986). Convexity and optimization in banach spaces. D. Riedel Publishing Company, Kluwer Academic Publishers Group, Dordrecht.

Delbaen, F. (2002). Coherent risk measures on general probability spaces. In: Advances in finance and stochastics: essays in honour of Dieter Sondermann (pp. 1-38). Springer-Verlag: Berlin. doi:10.1007/978-3-662-04790-3_1

Denis, L., \& Martini, C. (2006). A theoretical framework for the pricing of contingent claims in the presence of model uncertainty. Annals of
Applied Probability, 16, 827-852. doi:10.1214/105051606000000169

Dhaene, G., Goovaerts, M. J., Kaas, R., Tang, Q., Vanduffel, S., \& Vyncke, D. (2003). Solvency capital, risk measures and comonotonicity: A review. Research Report OR0416, Leuven: Catholic University of Leuven.

Gong, X. H. (1994). Connectedness of efficient solution sets for setvalued maps in normed spaces. Journal of Optimization Theory and Applications, 83, 83-96. doi:10.1007/BF02191763

Grechuk, B., Molyboha, A., \& Zabarankin, M. (2009). Maximum entropy principle with general deviation measures. Mathematics of Operations Research, 34, 445-467. doi:10.1287/moor.1090.0377

Grechuk, B., Molyboha, A., \& Zabarankin, M. (2011). Cooperative games with general deviation measures. Mathematical Finance, Forthcoming.

Jameson, G. (1970). Ordered linear spaces. Lecture notes in mathematics (Vol. 141). Berlin: Springer-Verlag.

Jaschke, S., \& Küchler, U. (2001). Coherent risk measures and gooddeal bounds. Finance and Stochastics, 5, 181-200. doi:10.1007/PL00013530

Kaina, M., \& Rüschendorf, L. (2009). On convex risk measures on $L^{p}$-spaces. Mathematical Methods of Operations Research, 69, $475-$ 495. doi:10.1007/s00186-008-0248-3

Konstantinides, D. G., \& Kountzakis, C. (2011). Risk measures in ordered normed linear spaces with non-empty cone-interior. Insurance: Mathematics and Economics, 48, 111-122. doi:10.1016/j.insmatheco.2010.10.003

Kountzakis, C. E. (2011). On efficient portfolio selection using convex risk measures. Mathematics and Financial Economics, 4, 223-252. doi:10.1007/s11579-011-0043-4

Kountzakis, C. E. (2011). Risk measures on ordered non-reflexive banach spaces. Journal of Mathematical Analysis and Applications, 373, 548-562. doi:10.1016/i.jmaa.2010.08.013

Kroll, Y., Levy, H., \& Markowitz, H. M. (1984). Mean-variance versus direct utility maximization. Journal of Finance, 39, 47-61. doi:10.1111/j.1540-6261.1984.tb03859.x

Kusuoka, S. (2001). On law invariant coherent risk measures. In: Advances in mathematical economics (Vol. 3, pp. 83-95). Tokyo: Springer. doi:10.1007/978-4-431-67891-5 4 4

Luenberger, D. G. (1969). Optimization by vector space methods. New York: John Wiley and Sons Inc.

Markowitz, H. (1952). Portfolio selection. Journal of Finance, 7, 7791.

Peng, S. (2007). G-expectation, G-brownian motion and related stochastic calulus of It $\hat{o}$ Type. In: stochastic analysis and applications, abel symposium (Vol. 2, pp. 541-567). Berlin: Springer.

Rockafellar, R. T., Uryasev, S., \& Zabarankin, M. (2003). Deviation measures in risk analysis and optimization. Research Report 2002-7, University of Florida.

Rockafellar, R. T., Uryasev, S., \& Zabarankin, M. (2006a). Generalized deviations in risk analysis. Finance and Stochastics, 10, 51-74. doi:10.1007/s00780-005-0165-8

Rockafellar, R. T., Uryasev, S., \& Zabarankin, M. (2006b). Master funds in portfolio analysis with general deviation measures. Journal of Banking and Finance, 30, 743-778. doi:10.1016/i.jbankfin.2005.04.004

Rockafellar, R. T., Uryasev, S., \& Zabarankin, M. (2006c). Optimality conditions in portfolio analysis with general deviation measures. $\mathrm{Ma}$ thematical Programming, 108, 515-540. doi:10.1007/s10107-006-0721-9

Rockafellar, R. T., Uryasev, S., \& Zabarankin, M. (2007). Equilibrium with investors with a diversity of deviation measures Journal of Banking and Finance, 31, 3251-3268. doi:10.1016/i.jbankfin.2007.04.002

Soner, H. M., Touzi, N., \& Zhang, J. (2011). A martingale representation theorem for the $G$-expectation. Stochastic Processes and their Applications, 121, 265-287. doi:10.1016/j.spa.2010.10.006

Tasche, D. (2002). Expected shortfall and beyond. Journal of Banking and Finance, 26, 1519-1533. doi:10.1016/S0378-4266(02)00272-8 


\section{E. KOUNTZAKIS}

Von Neumann, J. (1928). Zur theorie der gesellschaftsspiele. Mathematische Annalen, 100, 295-320. doi:10.1007/BF01448847

Xanthos, F. (2009). Conic characterizations of reflexive banach spaces.
Master's Degree Dissertation, Athens: National Technical University of Athens. 


\section{Appendix}

In this paragraph, we give some essential notions and results from the theory of partially ordered linear spaces which are used in the previous sections of this article.

Let $E$ be a (normed) linear space. A set $C \subseteq E$ satisfying $C+C \subseteq C$ and $\lambda C \subseteq C$ for any $\lambda \in \mathbb{R}_{+}$is called wedge. A wedge for which $C \cap(-C)=\{0\}$ is called cone. A pair $(E, \geq)$ where $E$ is a linear space and $\geq$ is a binary relation on $E$ satisfying the following properties:

1) $x \geq x$ for any $x \in E$ (reflexive).

2) If $x \geq y$ and $y \geq z$ then $x \geq z$, where $x, y, z \in E$ (transitive).

3) If $x \geq y$ then $\lambda x \geq \lambda y$ for any $\lambda \in \mathbb{R}_{+}$and $x+z \geq y+z$ for any $z \in E$, where $x, y \in E$ (compatible with the linear structure of $E$ ), is called partially ordered linear space.

The binary relation $\geq$ in this case is a partial ordering on $E$. The set $P=\{x \in E \mid x \geq 0\}$ is called (positive) wedge of the partial ordering $\geq$ of $E$. Given a wedge $C$ in $E$, the binary relation $\geq_{C}$ defined as follows:

$$
x \geq_{C} y \Leftrightarrow x-y \in C,
$$

is a partial ordering on $E$, called partial ordering induced by $C$ on $E$. If the partial ordering $\geq$ of the space $E$ is antisymmetric, namely if $x \geq y$ and $y \geq x$ implies $x=y$, where $x, y \in E$, then $P$ is a cone.

$E^{\prime}$ denotes the linear space of all linear functionals of $E$, while $E^{*}$ is the norm dual of $E^{*}$, in case where $E$ is a normed linear space.

Suppose that $C$ is a wedge of $E$. A functional $f \in E^{\prime}$ is called positive functional of $C$ if $f(x) \geq 0$ for any $x \in C$. $f \in E^{\prime}$ is a strictly positive functional of $C$ if $f(x)>0$ for any $x \in C \backslash\{0\}$. A linear functional $f \in E^{\prime}$ where $E$ is a normed linear space, is called uniformly monotonic functional of $C$ if there is some real number $a>0$ such that

$f(x) \geq a\|x\|$ for any $x \in C$. In case where a uniformly monotonic functional of $C$ exists, $C$ is a cone.

$C^{0}=\left\{f \in E^{*} \mid f(x) \geq 0\right.$ for any $\left.x \in C\right\}$ is the dual wedge of $C$ in $E^{*}$. Also, by $C^{00}$ we denote the subset $\left(C^{0}\right)^{0}$ of $E^{* *}$. It can be easily proved that if $C$ is a closed wedge of a reflexive space, then $C^{00}=C$. If $C$ is a wedge of $E^{*}$, then the set $C_{0}=\{x \in E \mid \hat{x}(f) \geq 0$ for any $f \in C\}$ is the dual wedge of $C$ in $E$, where $: E \rightarrow E^{* *}$ denotes the natural embedding map from $E$ to the second dual space $E^{* *}$ of $E$. Note that if for two wedges $K, C$ of $E \quad K \subseteq C$ holds, then $C^{0} \subseteq K^{0}$.

If $C$ is a cone, then a set $B \subseteq C$ is called base of $C$ if for any $x \in C \backslash\{0\}$ there exists a unique $\lambda>0$ such that $\lambda x \in B$. The set $B_{f}=\{x \in C \mid f(x)=1\}$ where $f$ is a strictly positive functional of $C$ is the base of $C$ defined by $f$. $B_{f}$ is bounded if and only if $f$ is uniformly monotonic. If $B$ is a bounded base of $C$ such that $0 \notin \bar{B}$ then $C$ is called well-based. If $C$ is well-based, then a bounded base of $C$ defined by a $g \in E^{*}$ exists. If $E=C-C$ then the wedge $C$ is called generating, while if $E=\overline{C-C}$ it is called almost generating. If $C$ is generating, then $C^{0}$ is a cone of $E^{*}$ in case where $E$ is a normed linear space. Also, $f \in E^{*}$ is a uniformly monotonic functional of $C$ if and only if

$f \in \operatorname{int} C^{0}$, where int $C^{0}$ denotes the norm-interior of $C^{0}$. If $E$ is partially ordered by $C$, then any set of the form $[x, y]=\left\{r \in E \mid y \geq_{C} r \geq_{C} x\right\}$ where $x, y \in C$ is called orderinterval of $E$. If $E$ is partially ordered by $C$ and for some $e \in E, E=\cup_{n=1}^{\infty}[-n e, n e]$ holds, then $e$ is called order-unit of $E$. If $E$ is a normed linear space then if every interior point of $C$ is an order-unit of $E$. If $E$ is moreover a Banach space and $C$ is closed, then every order-unit of $E$ is an interior point of $C$.

The partially ordered vector space $E$ is a vector lattice if for any $x, y \in E$, the supremum and the infimum of $\{x, y\}$ with respect to the partial ordering defined by $P$ exist in $E$. In this case $\sup \{x, y\}$ and $\inf \{x, y\}$ are denoted by $x \vee y$, $x \wedge y$ respectively. If so, $|x|=\sup \{x,-x\}$ is the absolute value of $x$ and if $E$ is also a normed space such that $\|x\|=\|x\|$ for any $x \in E$, then $E$ is called normed lattice.

Finally, we remind that the usual partial ordering of an $L^{p}(\Omega, \mathcal{F}, \mu)$ space, where $(\Omega, \mathcal{F}, \mu)$ is a probability space is the following: $x \geq y$ if and only if the set

$\{\omega \in \Omega: x(\omega) \geq y(\omega)\}$ is a set lying in $\mathcal{F}$ of $\mu$-probability 1 .

All the previously mentioned notions and related propositions concerning partially ordered linear spaces are contained in (Jameson, 1970).

A topological linear space $E$ is $E$ is boundedly order complete if for every bounded increasing net in the space $X$, the supremum of the elements of it exists. A cone $P$ of a linear topological space $E$ is called Daniell cone if every increasing net of $E$ which is upper bounded converges to its supremum.

Note that every well-based cone in a Banach space which has a base defined by a continuous linear functional. Every closed, well-based cone in a Banach space is a Daniell cone. Every Banach space partially ordered by a closed, well-based cone is a boundedly order-complete space.

A subset $F$ of a convex set $C$ in $L$ is called extreme set or else face of $C$, if whenever $x=a z+(1-a) y \in F$, where $0<a<1$ and $y, z \in C$ implies $y, z \in F$. If $F$ is a singleton, $F$ is called extreme point of $C$.

A family of cones in normed linear spaces having non-empty cone-interior are the Bishop-Phelps cones, also mentioned in (Konstantinides \& Kountzakis, 2011). The family of these cones in a normed linear space $E$ is the following:

$$
K(f, a)=\{x \in L \mid f(x) \geq a\|x\|\}, f \in L^{*},\|f\|=1, a \in(0,1) .
$$

A proof for the existence of interior points in these cones is contained in p. 127 of (Jameson, 1970).

Another family of cones with non-empty interior is the family of Henig Dilating cones. These cones are defined as follows: Consider a closed, well-based cone $C$ in the normed linear space $E$, which has a base $B$, such that

$0 \notin \overline{B+\delta B(0,1)}$. Let $\delta \in(0,1)$ be such that

$$
2 \delta B(0,1) \cap B=\varnothing,
$$

where $B(0,1)$ denotes the closed unit ball in $E$. If

$$
K_{n}=\overline{\text { cone }}\left(B+\frac{\delta}{n} B(0,1)\right), n \in \mathbb{N},
$$

then $C \subseteq K_{n+1} \subseteq K_{n}, n \in \mathbb{N}, K_{n}$ is a cone for any $n \geq 2$, $C \backslash\{0\} \subseteq \operatorname{int}\left(K_{n}\right), n \geq 1$. About these cones, see for example see Lemma 2.1 in (Gong, 1994). For example, a Bishop-Phelps cone $C=K(f, a)$ in a reflexive space which is a well-based cone as the construction of the $K_{n}, n \in \mathbb{N}$ requires, provides a set of interior points $C \backslash\{0\}$ of the cone $K_{n}, n \geq 1$. If we 


\section{E. KOUNTZAKIS}

consider the base $B_{f}=\{x \in C \mid f(x)=1\}$ defined by $f$, this base is a closed set where $0 \notin B_{f}$. Hence there is a

$g \neq 0, g \in E^{*}$ such that $g(y) \geq \delta^{\prime}>0$ for any $y \in B_{f} \cdot g$ can be selected to be such that $\|g\|=1$, hence

$\|y\| \geq g(y) \geq \delta^{\prime}>0$. By setting $\delta^{\prime}=2 \delta$, we may construct a sequence of approximating cones $K_{n}, n \in \mathbb{N}$, since we can set $g=f, \quad \delta^{\prime}=a \in(0,1)$. We remind that if $D$ is a convex set, then the set cone $(D)=\left\{x \in E \mid x=\lambda d, d \in D, \lambda \in \mathbb{R}_{+}\right\}$is a

wedge and by $\overline{\text { cone }}(D)$ we denote its norm (or weak) closure. 